\title{
Therapeutic efficacy of nanoparticles and routes of administration
}

\author{
Dhrisya Chenthamara', Sadhasivam Subramaniam ${ }^{1,2^{*}}$, Sankar Ganesh Ramakrishnan ${ }^{1}$ Swaminathan Krishnaswamy ${ }^{1}$, \\ Musthafa Mohamed Essa ${ }^{3^{*}}$, Feng-Huei Lin ${ }^{4}$ and M. Walid Qoronfleh ${ }^{5^{*}}$ (D)
}

\begin{abstract}
In modern-day medicine, nanotechnology and nanoparticles are some of the indispensable tools in disease monitoring and therapy. The term "nanomaterials" describes materials with nanoscale dimensions $(<100 \mathrm{~nm})$ and are broadly classified into natural and synthetic nanomaterials. However, "engineered" nanomaterials have received significant attention due to their versatility. Although enormous strides have been made in research and development in the field of nanotechnology, it is often confusing for beginners to make an informed choice regarding the nanocarrier system and its potential applications. Hence, in this review, we have endeavored to briefly explain the most commonly used nanomaterials, their core properties and how surface functionalization would facilitate competent delivery of drugs or therapeutic molecules. Similarly, the suitability of carbon-based nanomaterials like CNT and QD has been discussed for targeted drug delivery and siRNA therapy. One of the biggest challenges in the formulation of drug delivery systems is fulfilling targeted/specific drug delivery, controlling drug release and preventing opsonization. Thus, a different mechanism of drug targeting, the role of suitable drug-laden nanocarrier fabrication and methods to augment drug solubility and bioavailability are discussed. Additionally, different routes of nanocarrier administration are discussed to provide greater understanding of the biological and other barriers and their impact on drug transport. The overall aim of this article is to facilitate straightforward perception of nanocarrier design, routes of various nanoparticle administration and the challenges associated with each drug delivery method.
\end{abstract}

Keywords: Nanoparticles, Nanocarriers, Drug delivery, Drug administration, Targeted drug delivery, Administration route, Therapeutics, Cancer

\section{Background}

\section{Nanotechnology and nanoparticles}

In the Greek language, the words nano means "dwarf" and the SI prefix denotes $10^{-9}$ or 0.000000001 . By definition, nanotechnology is a fusion of advanced manufacturing science and engineering where the synthesis or assembly of material is aimed at the nanometer scale $(1-100 \mathrm{~nm})$ or one-billionth of a meter. The unique property of nanosized material as compared to bulk material is the advantage of

\footnotetext{
* Correspondence: sadhaon@gmail.com; sadhaofficial@buc.edu.in; drmdessa@gmail.com; wqoronfleh@qf.org.qa

'Department of Microbial Biotechnology, Bioprocess and Biomaterials Laboratory, Bharathiar University, Coimbatore, India

${ }^{3}$ Department of Food Science and Nutrition, College of Agricultural and Marine Sciences, Sultan Qaboos University, Muscat, Oman

${ }^{5}$ Research and Policy Department, World Innovation Summit for Health (WISH), Qatar Foundation, P.O. Box 5825, Doha, Qatar

Full list of author information is available at the end of the article
}

more surface to volume ratio. Nanoparticles (NPs) have wide-spread applications in various sectors ranging from agriculture to medicine. In medicine, nanoparticles are continuously being improved for drug delivery, screening of various diseases and tissue engineering, to name a few. Consequently, nanotechnology has begun playing a pivotal role in catalysis, energy and environment, agriculture, optics, sensors, computers and many others [1]. The current review explores the advancements in nanoparticlemediated targeted drug delivery along with discussing the efficacy and limitations of various administration routes. Besides conventional drugs, recombinant proteins, vaccines, and nucleotides may also be effectively delivered by NPs [2]. Nanoparticles can be synthesized from various organic or inorganic materials such as lipids, proteins, synthetic/natural polymers, and metals [3, 4]. Nanoparticles can be classified into several groups such as polymeric

(c) The Author(s). 2019 Open Access This article is distributed under the terms of the Creative Commons Attribution 4.0 International License (http://creativecommons.org/licenses/by/4.0/), which permits unrestricted use, distribution, and reproduction in any medium, provided you give appropriate credit to the original author(s) and the source, provide a link to the Creative Commons license, and indicate if changes were made. The Creative Commons Public Domain Dedication waiver (http://creativecommons.org/publicdomain/zero/1.0/) applies to the data made available in this article, unless otherwise stated. 
nanoparticles, liposomes, dendrimers, micelles and inorganic nanoparticles, based on the components used for synthesis or the structural aspects of the NP (Fig. 1). The fabrication methods and the properties of nanoparticles would also determine its application and utility. However, the type of nanoparticle used in the targeted delivery of therapeutics has its own positive and negative influences [3].

\section{Natural and synthetic polymer nanoparticles}

A wide range of polymer nanoparticles has been described owing to advancements in polymer science and nanotechnology. The unique property or desirable characteristics of polymeric nanoparticles decide its potential application. The most important properties of polymeric nanoparticles are biocompatibility and biodegradability. Therefore, they are widely used as a drug delivery system [5]. Besides, they must retain high stability in a biological environment. For drug delivery applications, the drug may either be encapsulated or immobilized on the polymer and subsequently released into the target site by diffusion or desorption [6]. Based on the drug-encapsulation method they are classified into three types. The first type consists of linear polymers (i.e., it uses a covalent approach for drug conjugation), the second category is labelled as polymeric micelles (formed by amphiphilic block copolymers) and the third group involves hydrogels (i.e., hydrophilic drug encapsulation) [7]. The main characteristic of the polymeric nanoparticle is the controlled release of therapeutic agents. Biodegradable polymeric nanoparticles are not only used as carriers for pharmaceutical drugs but also to deliver proteins and DNA. Synthetic polymers such as polylactide-polyglycolide copolymers, polyacrylates and polycaprolactones (PCL), polylactic acid (PLA), poly (lactic-co-glycolic acid) (PLGA) are often used in nanoparticle synthesis. The tissue compatibility nature of PLA and PLGA make them useful in controlled release formulation for parenteral and implantation drug delivery applications [8]. The structural properties of polysaccharide nanoparticles are determined by their chemical composition [9].

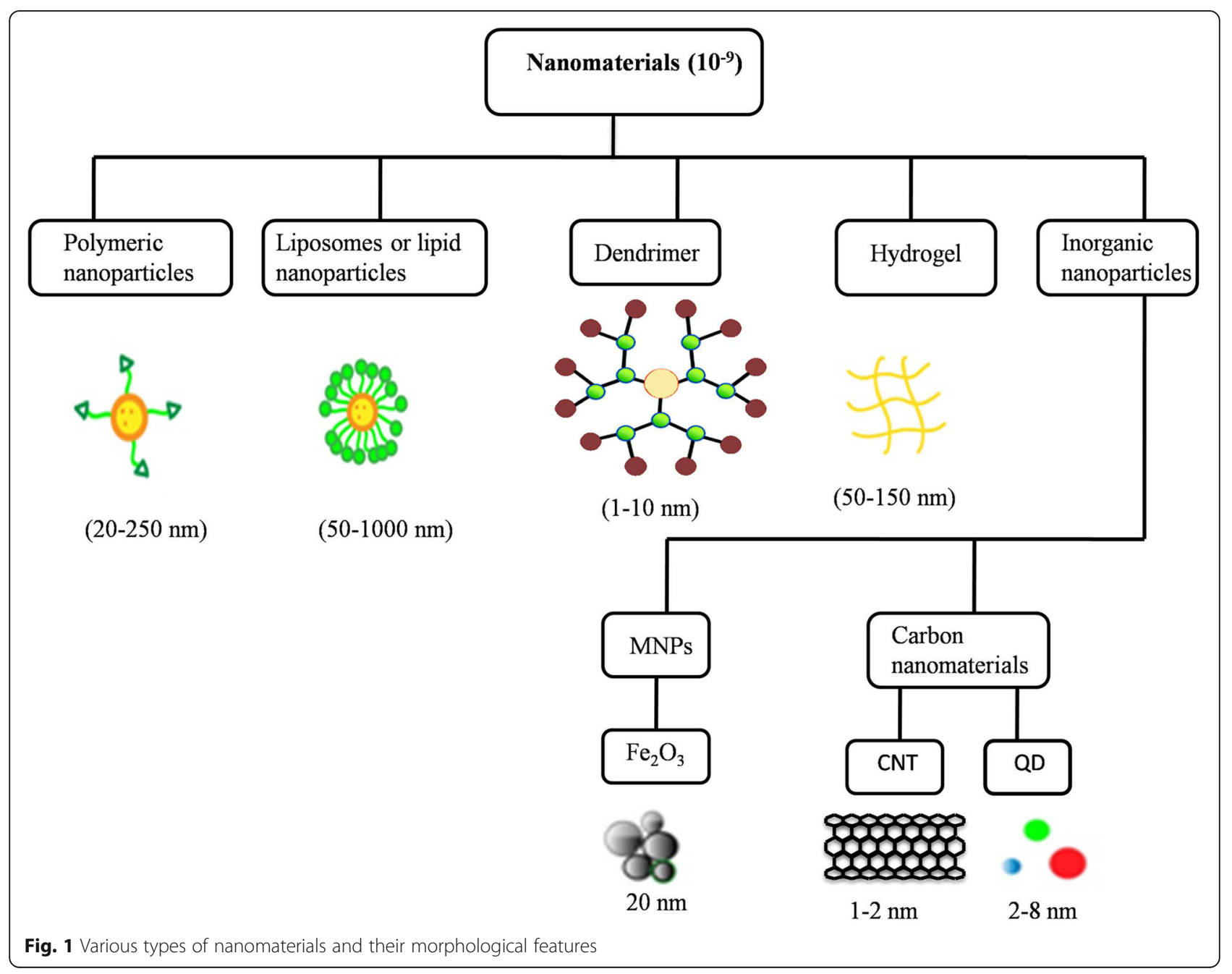


Polysaccharides are a substantial component of natural polymers and are mainly derived from algae (e.g., alginate), plant (e.g., pectin \& guar gum), microbial (e.g., dextran \& xanthan gum), and animal (chitosan\& chondroitin) products. Synthetic polymer nanoparticles are preferred over natural polymeric nanoparticles for sustained release [10]. These polymers have exceptional material properties because of their chemical structure and type of functional group(s). Moreover, they can also be altered based on the method of synthesis. The advantages of synthetic polymeric nanoparticle include easy fabrication and absence of biological contamination. Polycationic polymers have shown better mucoadhesive properties and, as a result, are widely used in mucoadhesive drug delivery [11]. Chitosan is mucoadhesive and soluble only at acidic $\mathrm{pH}$. Hence, chemical modification of chitosan is being carried out to enhance its mucoadhesive properties. Chitosan derivatives like trimethyl chitosan (TMC), thiolated chitosan, chitosanethylenediaminetetraacetic acid, etc. have showed improved solubility and mucoadhesive properties [12]. Sajomsang et al have synthesized two methylated derivatives of chitosan and found that increasing the degree of quaternization will lead to stronger mucin-particle interaction [13]. Thanou et al reported the ability of TMC to enhance the permeation of the peptide drug buserelin, a gonadotropinreleasing hormone agonist, across intestinal epithelia in vitro (Caco- 2 cell monolayers) and in vivo (rats) [14]. Gatti et al prepared nanoparticles based on chitosan/dextran sulfate formed by polyelectrolytes condensation for insulin delivery. The encapsulation prevented insulin from partial degradation and displayed sustainable release indicating efficient mucus complexation between mucin and nanoparticles [15].

Polymer-coated nanoparticles have been used to improve the biodistribution kinetics. The nanoparticle surface coated with polyethylene glycol (PEG) has increased blood drug concentration in the brain, kidney, and intestine by evading the reticuloendothelial clearance system [16]. The bio-inert characteristic of the PEG polymer is a classic example of the preparation of cytocompatible multifunctional polymeric nanoparticle and surface modification. The foremost desirability of PEGylation for drug delivery lies in its ability to extend their stability in the mucous and to reduce the nanoparticle clearance by the immune cells [17]. The unique architecture of nanosized carriers considerably overcomes the limitation of conventional drug delivery methods and has an impact on advanced therapy for various diseases like tuberculosis and pulmonary hypertension [3].

\section{Poly (lactic-co-glycolic acid) (PLGA)}

Among the synthetic polymers, poly (lactic-co-glycolic acid) PLGA (obtained by the condensation of lactic acid and glycolic acid) is considered a base material for numerous biomedical applications. The main appeal of PLGA NPs can be attributed to the fact that they are hydrolyzed into their monomeric units such as lactic acid and glycolic acid, which are byproducts of various metabolic pathways in the body under normal physiological conditions [18]. Technological sophistication has enabled PLGA nanoparticles to be explored not only to encapsulate anticancer drugs, diabetic medications or hormones but they also offer a platform for multifunctional imaging in cancer diagnostics [5]. One of the lures of using PLGA in medical devices (e.g., orthopedic fixation devices) or nanoparticle fabrication is that the rate of biodegradation can be controlled by adjusting its molecular weight (MW) or copolymer ratio [19]. The US Food and Drug Administration (FDA) and the European Medicine Agency (EMA) have permitted the use of PLGA for drug delivery applications in humans [20]. Despite PLGA having minimal toxicity, their acidic nature does not favor the release of acid-labile drugs. However, it could be revamped by formulating a suitable mix with carbohydrate polymers like chitosan, alginate, and poly (isoprene), etc. The combination of hydrophobic or amphiphilic polymers such as PLGA and PLGA-PEG offers great promise in drug delivery, but the applied experimental conditions like sonication could affect the stability of the drug molecule encapsulated within. Encapsulation of a range of anticancer drugs, namely doxorubicin, paclitaxel, dexamethasone, cisplatin 5-fluorouracil and 9-nitrocamptothecin, have been reported as using PLGA nanoparticles [21]. The PLGA microsphere has successfully protected the encapsulated DNA from nuclease degradation [22] and to attain a stable gene expression, the encapsulated DNA in PLGA has to undergo sustained release after intracellular uptake and endolysosomal escape [23]. To improve the efficiency of PLGA nanoparticle as a drug delivery system, zinc (II) phthalocyanine $(\mathrm{ZnPc})$ was incorporated to increase the rate of permeation and tissue uptake for the photodynamic activity in mice [24]. Likewise, the functionalization of PLGA with polyethyleneimine (PEI) was shown to be effective in siRNA delivery. The existing evidence suggested that PLGA is one of the most successful in vivo biodegradable drug delivery systems owing to its simple hydrolysis degradation mechanism [24].

\section{Chitosan}

There are numerous polymers that have been approved for biomedical applications. Among them, chitosan is the most important naturally occurring cationic polymer approved by the US FDA and EMA for tissue engineering, drug delivery and also gene delivery [25]. Mumper et al first reported the use of chitosan for in vitro gene delivery [26]. Hydrophobic polymers such as PLGA have a serious limitation in delivering macromolecules across the biological surfaces. Hence, 
colloidal hydrophilic polymers are the primary choice for delivering such macromolecules effectively. Through different mechanisms like ionic crosslinking or complexation and desolvation, chitosan is capable of forming colloidal nanoparticles which can protect the macromolecule of interest [27]. The excellent biocompatible and biodegradable nature of chitosan makes it useful in various drug delivery applications. The structure of chitosan is highly favorable for effortless functionalization with its primary hydroxyl and amino groups that also improve the physical and biological properties of chitosan during the conjugation process. The hydrophilic nature of chitosan aids an easy conjugation of hydrophobic moiety which in turn leads to the formation of self-assembled nanoparticles that are useful for targeted drug delivery applications [28]. Because of their effortless functionalization and mucoadhesive properties, chitosan-based delivery systems have been the most studied and demonstrated platform for delivering drugs or pharmaceuticals to various organs. Chitosan capsules were designed to enhance the localization of 5-Aminosalicylic acid (5ASA) for colon-specific drug delivery (Fig. 2) [28, 29]. An affinity-based interaction between the hydroxyl and amino groups of chitosan and hydroxyl groups of dexamethasone has suggested that chitosan-films are useful for sustained release of dexamethasone [30]. Low molecular weight chitosan (LMWC) (19 and $31 \mathrm{kDa}$ ) are promising drug carriers (e.g., LMWC with prednisone) for renal or kidney targeting [31].

\section{Liposomes and solid lipid nanoparticles (SLNP)}

The use of lipid-based nanoparticles was initially derived from the biocompatible concept, where the tiny lipid cholesterol molecules and phosphatidylcholine are popular [32]. Another reason for using lipid-based nanoparticles is their easy cellular uptake of drugs because of the outer lipid bilayer [3,33]. Two of the most important lipid-based nanomaterials are liposomes and solid lipid nanoparticles. Liposomes consist of a lipid bilayer enclosing an aqueous core while lipid NP consists of lipid monolayer enclosing a solid lipid core [34]. While they are slightly different in their structure, both can be effectively used in drug delivery applications. Liposomes and solid-lipid nanoparticles are particularly considered effective in inhalation therapy for chronic lung diseases since they are stable during aerosolization [33]. The effectiveness of SLNPs was demonstrated by SLNPs loaded berberine (benzylisoquinoline alkaloid) which showed better bioavailability and increased the antidiabetic effect in a diabetic mouse model [35]. A phytocompound Aloe-emodin, an anthraquinone, loaded in SLNPs displayed increased anticancer effect in hepatoma and breast cancer cell lines [36]. Baeck et al noted increased bioavailability of

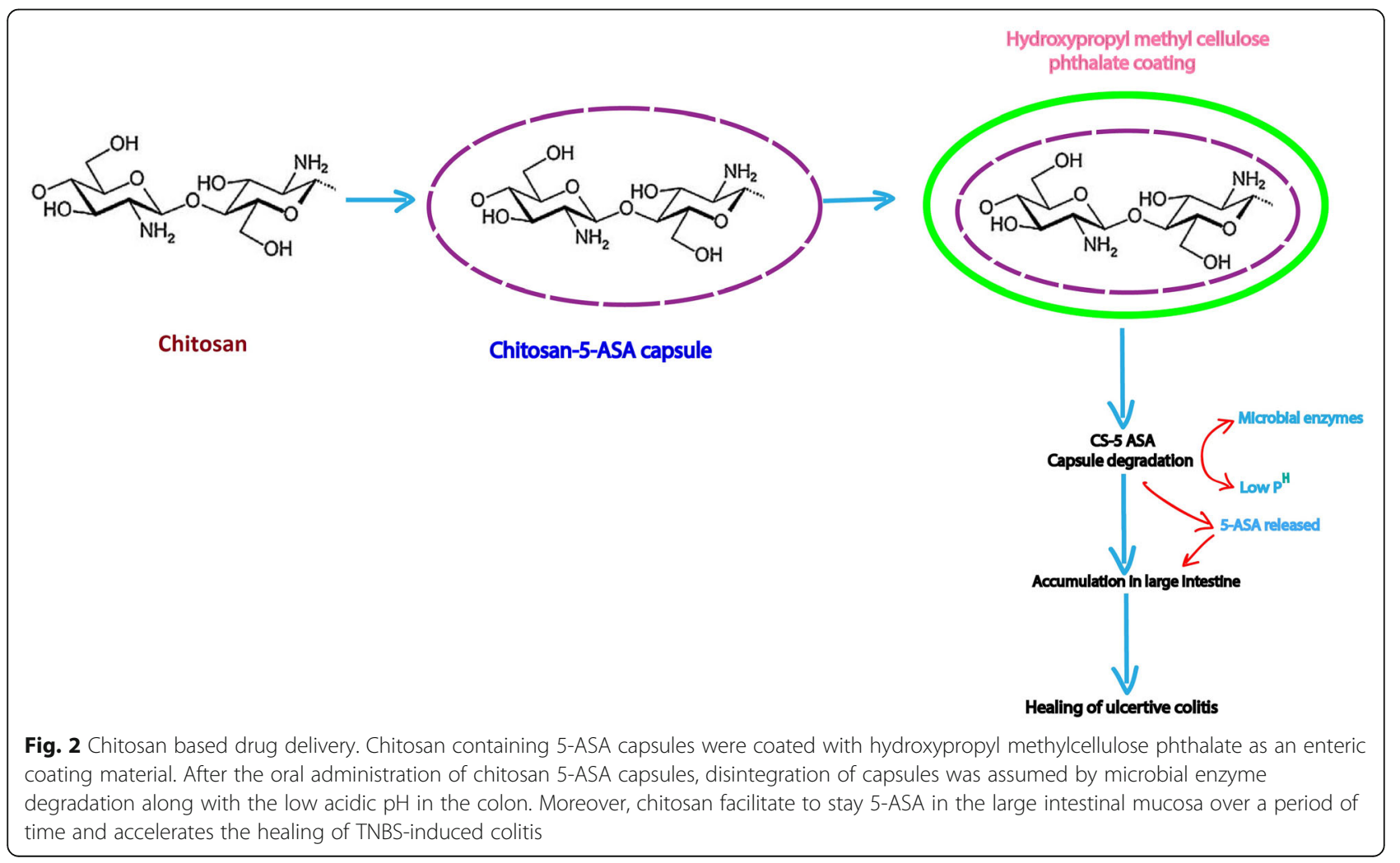


curcumin in lymphatic cells when loaded with $\mathrm{N}$ carboxymethyl chitosan-coated SLNPs [37].

\section{Dendrimers}

Dendrimers are synthetic, well-defined and highly mono-dispersed symmetric molecules which have a repetitive branched pattern. They can demonstrate better physicochemical and rheological properties as compared to conventional linear polymers. Regardless of the advancements in dendrimer research, the use of dendrimers as drug carriers is still poorly translated into the clinical application [38]. Although it shows its excellence as drug and gene delivery agents, dendrimers can display cytotoxic and hemolytic properties, raising potential toxicity safety concerns. As dendrimers are nondegradable in the physiological environment, it results in serious side effects induced by the accumulation of nondegradable artificial macromolecules inside the cells or in the tissues. The cationic characteristics of these polymers result in an interaction with the negatively charged cell membranes, thereby causing cell destabilization with the leakage of cytoplasmic proteins and subsequent lysis $[39,40]$. Moreover, the size and surface functionality of the final formulation is precisely controllable [41]. The surface of PEGylated dendrimers may have higher drug load than the unmodified dendrimers and is designed to escape the body's defense actions and circulate in the blood for an extended period of time. A variety of drugs or therapeutic molecules can be encapsulated in dendrimers using a simple electrostatic interaction or covalent attachment [42-44]. The polyvalency and strong spatial distribution of multiple functionalities on the surface of the dendrimer are major assets making them a desirable agent for combating cancer, inflammation, HIV, etc. along with drugs and gene delivery [45]. The surfacemodified dendrimers by lauroyl chains and PEG-2000 have significantly reduced cytotoxicity in Caco-2 cells [46]. Likewise, newer PEGylated polyamidoamine (PAMAM) dendrimers (4.0 G PAMAM) synthesized by Michael addition and amidation reactions were used for the delivery of the anticancer drug 5-fluorouracil which reduced the rate of drug release and hemolytic toxicity [47]. Acetylation of PAMAM dendrimers is reported to be a promising siRNA delivery agent again because of reduced cytotoxicity [48]. Several new dendrimers poly (propylene imine) (PPI), poly (amidoamine) (PAMAM), and poly(L-lysine) (PLL) (i.e., PEGylated PLL dendrimer with docetaxel) are in clinical trials owing to their well-defined architecture and facile surface tailoring [49]. These results substantiate a positive indication of dendrimers potential in nanotechnology-based cancer therapy.

Synthesis of multifunctional dendrimers for theranostic applications is a contemporary research direction. One of the emerging applications of dendrimers is focused on cancer theranostics. Differently sized macromolecular and nanosized dendrimer MRI contrast agents have been reported for various applications as they provide sufficient contrast enhancement [50-52]. The approved MRI contrasting agents are of low molecular weight (e.g. gallium) hence they will be easily degraded and eliminated by the renal infiltration system. Dendrimer-conjugated contrasting agents display prolonged blood circulation time [52]. A multifunctional PAMAM dendrimer was used as a template to encapsulate gadolinium oxide nanoparticles $\left(\mathrm{Gd}_{2} \mathrm{O}_{3} \mathrm{NPs}\right)$ for enhancement in vivo magnetic resonance imaging [53]. The PAMAM- $\mathrm{Gd}_{2} \mathrm{O}_{3}$ nanoparticles exhibited a longer longitudinal relaxation time (T1) and better biocompatibility than the clinically popular Gd-DTPA contrasting agents. PAMAM dendrimers coated with magnetite nanoparticles $\left(\mathrm{Fe}_{3} \mathrm{O}_{4}\right)$ are reported as successful nanoplatforms for combined therapeutic and diagnostic purposes with excellent contrasting properties in MRI [54]. Design and development of such multifunctional model systems has significant potential in anticancer therapy.

\section{Hydrogel}

Hydrogels are three-dimensional polymeric networks and contain greater than $90 \%$ of water because of its hydrophilic nature. Biopolymers like chitosan and hyaluronic acid (HA) are the top-line macromolecules used for cancer therapy and imaging. Hydrogel fabrication techniques and usage are increasingly common for pharmaceutical and biomedical applications (Fig. 3) [55-59]. Chitosan-based hydrogels are in absolute demand for drug-delivery applications. Intelligent hydrogels are classified under smart biomaterials because the sensitivity and application of such hydrogels are regulated by external stimulus of temperature, $\mathrm{pH}$, photo and magnetic factors [60]. Photosensitive azidehydroxyethyl chitosan (AZ-HECTS) synthesized by UV radiation has shown biodegradable and biocompatible property with sustained heparin release [61]. A redox-responsive supramolecular hydrogel is a kind of smart or intelligent hydrogel that has been described for the successful delivery of 10-hydroxy camptothecin (HCPT) peptide as a potential anticancer agent [62]. Temperature-sensitive hydrogel like poly(N-isopropyl acrylamide), pNIPA, has significant interest in drug delivery and it exhibits volume phase transition at $32^{\circ} \mathrm{C}$. Below this temperature, water soluble drugs can be encapsulated and the amide groups initiated hydrogen bonds in pNIPA hydrogels are cleaved above $32{ }^{\circ} \mathrm{C}$, resulting in controlled drug release [63]. $\mathrm{pH}$ sensitive hydroxyethylacryl chitosan (HC) and sodium alginate (SA) hydrogel were reported for the drug paracetamol under in vitro conditions [64].

\section{Nano hydrogel}

It is known that chronic inflammation is strongly tied to the initiation and progression of cancer. Hence, an anionic polysaccharide gellan gum based nanohydrogel was 


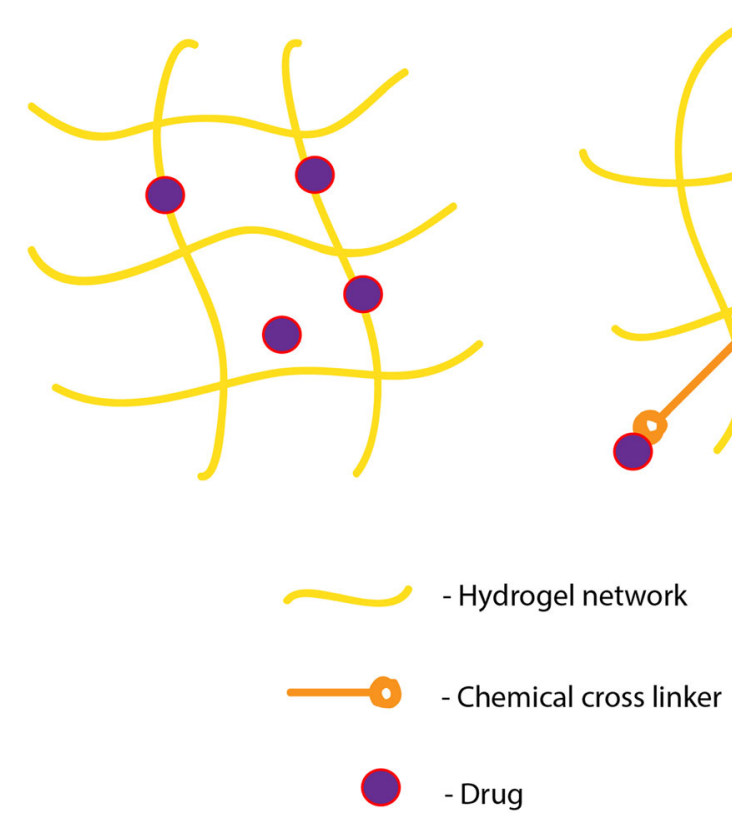

Fig. 3 Formulation of hydrogel-drug matrix. The most routinely followed strategy for drug delivery from the hydrogel matrix is physical or chemical interactions. In physical interactions, the affinity between the gel and drug is often charge based. If the gel matrix is having more amino functional groups it could be useful for the delayed release of anionic drugs. Simply, the polymers can have significant effect on prolonged release of drugs of opposite charge. As opposed to physical interaction, drug is permanently linked to hydrogel matrix via covalent crosslinks. This kind of binding could be achieved with other methods like UV irradiation and redox-responsive supramolecular assembly

developed to offer the dual benefits of anti-inflammatory and anticancer features by chemically cross-linking glucocorticosteroid prednisolone and physically encapsulating paclitaxel [65]. The nanohydrogel system has offered synergistic drug effect from the incorporated drugs by facilitating solubility, drug-uptake and targeted tumorigenesis inhibition via attacking inflammatory components and malignant cells. Systemic chemotherapy is still a preferred first line treatment for solid tumors as it offers effective therapeutic drug load to cancer cells, prolonging drug activity and decreasing the side effects to normal cells. 5-Fluorouracil (5-FU) has been classified as an anti-metabolite with anti-neoplastic activity but having the disadvantage of poor half-life $(16 \mathrm{~min})$ and being rapidly metabolized by dihydropyrimidine dehydrogenase. The drawbacks of direct administration of 5 -FU is proposed to be greatly reverted by the thermosensitive methylcellulose nanohydrogel containing 5-FU and it could be used as an effective systemic chemotherapy for solid tumors such as head and neck cancers, colorectal cancer and brain tumor [66].

\section{Inorganic nanoparticles}

Inorganic nanoparticles exhibit different material properties and hence have many potential applications. The optical and magnetic properties of inorganic nanoparticles have paved way into their usage in cancer therapies. They also exhibit features such as fluorescence, near- infrared (NIR) absorption and Raman enhancement making them extremely useful in image-guided therapies. Inorganic nanoparticles derived from their macromolecule counterparts such as iron oxide, gold or silica have emerged as highly valuable building blocks. Owing to their multifunctional properties, inorganic nanoparticles (gold and iron oxide) were found to be suitable in computed tomography (CT), surface plasmon resonance (SPR), magnetic resonance imaging (MRI), or positron emission tomography (PET) as image contrast agent [67]. Accordingly, the scope of inorganic nanoparticle in image-guided early disease screening has vastly improved. Similarly, the term "multi-modal imaging" has become recently popular as it offers two or more imaging techniques to retrieve more information and permit an effective treatment plan. The multifunctional nanoparticle system containing Prussian blue (PB), serum albumin (BSA), and indocyanine green (ICG) was reported as a novel theranostic agent since it could provide dual-mode magnetic resonance (MR) and nearinfrared (NIR) fluorescence imaging in photothermal and photodynamic (PTT-PDT) therapy [68]. Gold (Au) capped magnetic core/mesoporous silica shell nanoparticles were fabricated to obtain the synergistic effect of combined photothermal/chemo-therapy and multimodal imaging in a single system [69]. Nanoparticles made of $\mathrm{Au}$ or $\mathrm{Ag}$ conjugated with polyethyleneimine (PEI) have also been used to deliver genes [70]. Functionalization of 
$\mathrm{Au}$ NPs with PEG and coumarin were found to efficient incorporation capacity into breast cancer cells without any observed toxicity to other normal cells. A major limitation of using inorganic nanoparticles is that their long-term toxicity and clearance have not been evaluated sufficiently [71].

\section{Magnetic nanoparticles (MNPs)}

MNPs are distinctively different from other typical nanoparticles due to their unique magnetic property. The main limitations of MNPs are burst drug release and low stability features. To overcome this issue, surface ligands are attached to MNPs, which in turn improve the stability and solubility in biological environments along with exhibiting lesser side effects [72]. Owing to the MNPs large-surfaceto-volume ratio, it offers numerous chemically active-sites for biomolecule conjugation (Fig. 4). Thus, it provides longer circulation time, target-specific binding and drug delivery [73]. As of now, chemotherapy, radiotherapy, and medical procedures are considered the three clinically accessible treatments in tumor management. The main drawbacks of these treatments are the side effects as they are not specific. As an alternative to this, thermotherapy is being used to kill a tumor cell with principles based on the higher themo-sensitive nature of cancer cells than normal cells. This can be achieved by hyperthermia in which the temperature of a local region or the body is increased up to $40-45^{\circ} \mathrm{C}$ through radiation. The second method, thermo-ablation, uses above $45^{\circ} \mathrm{C}$ temperatures to the diseased area to destroy tissues. In animal models, MNPmediated hyperthermia has been successfully used for the treatment of mice tumors [74]. Their magnetic property is not only useful in magnetic separation and magnetic resonance imaging but also useful in many applications; namely tissue engineering, gene transfection, magnetic memory devices, and magnetic ink, etc. The application of MNPs can also be extended to drug targeting and cell sorting [75]. Paclitaxel (PTX) or rapamycin loaded glycerol mono-oleate-coated magnetic nanoparticles (GMOMNPs) conjugated human epidermal growth factor receptor 2(HER2) antibody showed 24 times more effective anticancer activity than the free drug [76]. The potential

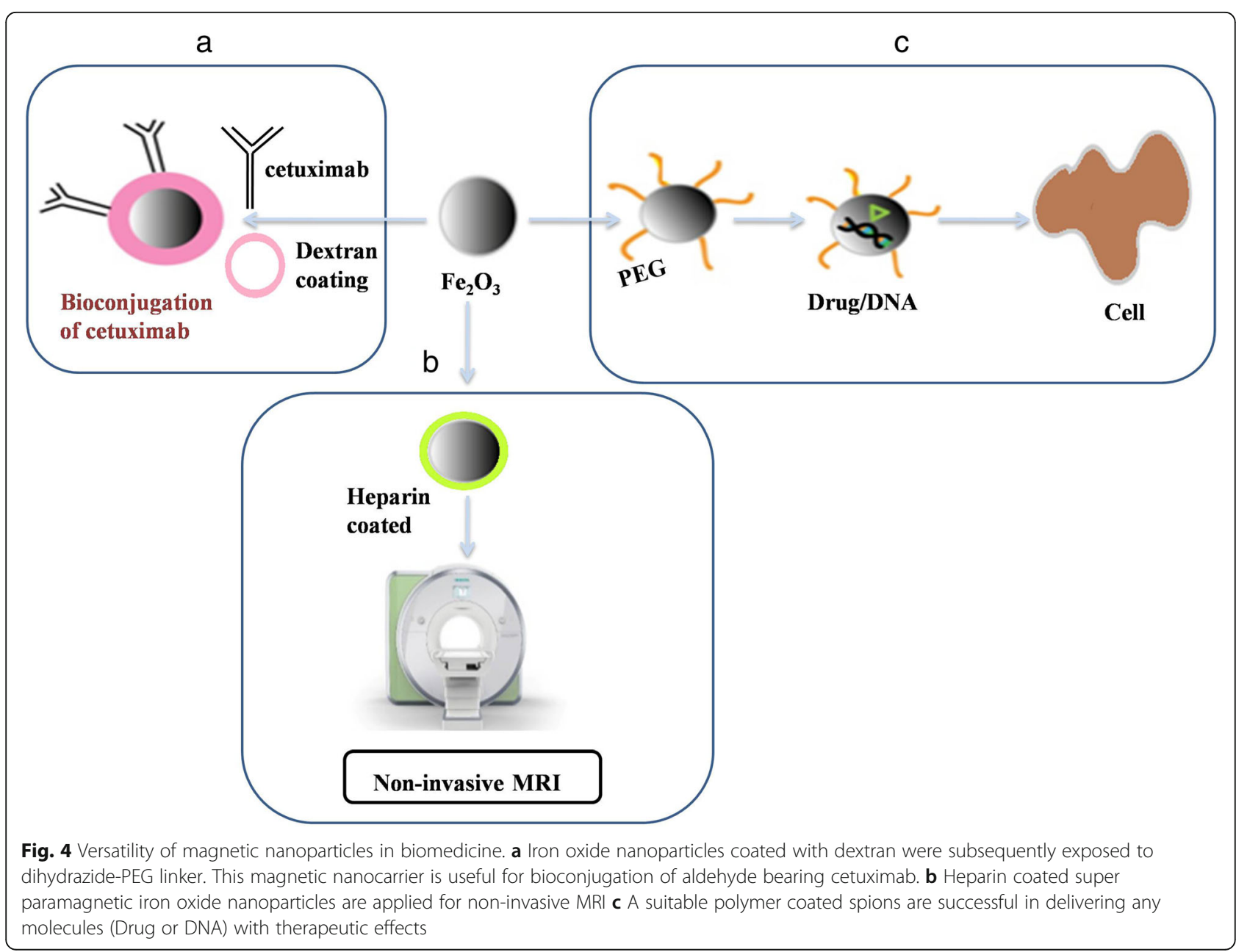


for nanotoxicity exists and presents a great concern; yet research in this fascinating area continues.

\section{Carbon nanotubes}

Carbon nanotubes (CNTs) are defined by the hexagonal arrangement of carbon atoms that leads to cylindrical nanostructure formation. Arc discharge, laser ablation, and chemical vapor deposition are some of the imperative methods for the production of CNTs. Graphene sheets are rolled at certain angles to create desired CNTs and the said nanotubes are either classified as single wall (SWCNT) or multi-wall (MWCNT) depending on the layer of graphene sheets. The outer diameter of SWCNT is typically between 0.4 and $2.0 \mathrm{~nm}$ and between 10 and $100 \mathrm{~nm}$ for MWCNTs [77]. CNTs have unique electrical, mechanical and optical properties along with a high surface area that make them appropriate for attaching biological cargoes. Originally, CNTs were toxic because of their hydrophobic surface and limited aqueous solubility. As a result, CNT mediated the following harmful effects by free radical formation, reactive oxygen species (ROS), apoptosis, granuloma formation, and increased inflammatory responses. This toxicity of CNTs can be overcome by proper functionalization methods. The structural feature of CNT is better utilized for changing the surface of the CNT, i.e., the inner hollow structure is used to accommodate suitable drugs and the outer surface is modified via physical or chemical bonding [78]. The CNT surface can be customized with molecules of choice by adsorption, electrostatic interaction or covalent bonding that render them hydrophilic [79].
Multi-functionalization strategy is an interesting concept wherein CNT can be functionalized with a fluorescent probe and amphotericin B to examine cellular uptake and controlled drug delivery. Surface engineered CNTs are taken up into cells by endocytosis, phagocytosis or membrane translocation; however, certain properties like tube dimensions, surface functionalization and the cell type determine the uptake rate [80]. Higher drug loading on the surface or inner core, ease of conjugation with ligands, thermal ablation and easy cellular uptake are attractive $\mathrm{CNT}$ features in cancer treatment and diagnostics [81]. They can target deliver anticancer drugs to arrest cancer cells progression. CNTs have also been used to carry topoisomerase I inhibitors (topotecan) and topoisomerase II inhibitors (teniposide) to slow the growth of cancer cells down by inhibiting DNA topoisomerase activity [82]. Similar to nanomaterials drug delivery, CNTs have been used in transfection for delivering genes or DNA to mammalian cells. Recently, siRNA based therapy was found to be attractive for the treatment of various diseases including cancer. However, siRNAs are prone to easy degradation by RNases, henceforth effective strategies are requisite for delivering the siRNA molecules. Non-covalently functionalized SWC NTs by PEI conjugated to 1,2-distearoyl-sn-glycero-3phosphoethanolamine- $\mathrm{N}$-[amino (polyethylene glycol)2000] (DSPE-PEG-PEI) were successful in facilitating siRNA delivery in vitro as well as in vivo (Fig. 5) [83]. CNTs were also used in neuron-repair strategies or neuro-tissue engineering as nerve tissue reconstructing platforms [84]. They could act as an electrical interface
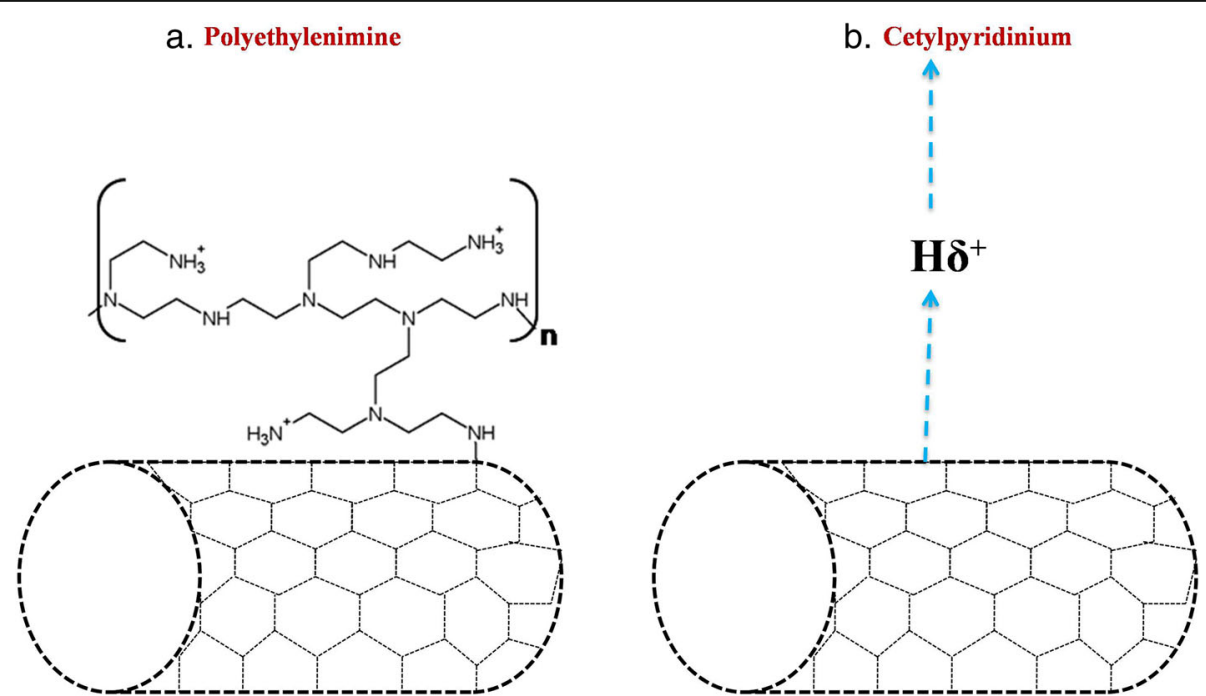

Fig. 5 CNT functionalization for siRNA delivery. To achieve an effective siRNA delivery, CNTs were functionalized with covalent and non-covalent crosslinking. a CNT covalently linked with cationic polymer polyethylenimine (PEI) b CNT functionalized with non-covalent interaction with cationic cetylpyridinium. The different functionalization methods were tried to achieve efficient gene silencing. A thin and long structural feature of CNT offers long surface area and nano-needle morphology facilitates easy translocation over the plasma membrane via endocytosis-independent pathway 
for neuronal stimulation, recording [85] both in vitro and in vivo, and also promote neuronal survival, differentiation, growth, and performance [86].

\section{Quantum dots}

Quantum dots (QDs) are nanosized semiconductor particles $(2-10 \mathrm{~nm})$ prepared from chalcogenides (selenides or sulfides) of cadmium or zinc. In general, the size and shape of the quantum dots will determine its optoelectronic properties. Longer quantum dots (radius of $5-6 \mathrm{~nm}$ ) will emit orange or red color and the smaller QDs (radius of 2-3 nm) emit the colors blue and green [87]. From an application perspective, QDs are prepared like core-shell structures with an appropriate functional coating through a high-temperature strategy which yielded particle size of $<10 \mathrm{~nm}$ with narrow size distribution [17]. The versatile surface chemistry and photo-physical property allow the preparation of multifunctional QDs for drug loading, targeting, controlled release, and monitoring of pharmacokinetics and biodistribution [74]. Multifunctional nanocomposite, i.e., carboxyl modified, QDs are crosslinked with amino-functionalized immune-liposomes. These are prepared with anti-human epidermal growth factor receptor 2 (anti-HER2) scFv for cancer diagnostics and targeted therapeutics in HER2 overexpressing human breast carcinoma cells, SK-BR-3 and MCF7-C18 (Fig. 6) [88]. With further technological advances, fluorescent carbon quantum dots (CQDs) have emerged as a potential entrant to traditional semiconductor quantum dots. As quantum dots, CQDs have been used in sensing, imaging and medicinal applications [89].

\section{Physical properties of nanoparticle Optical property}

Noble metal nanoparticles $(\mathrm{Cu}, \mathrm{Ag}$, and $\mathrm{Au})$ are known for their unique optical properties exhibited near UV and visible spectral wavelength range. Furthermore, the optical properties of such nanoparticles are used to attain desired contrast in various cell imaging applications. Gold nanoparticles (AuNPs) are extensively used in optical imaging due to their unique plasmonic properties, hence AuNPs-assisted bioimaging is mainly used in

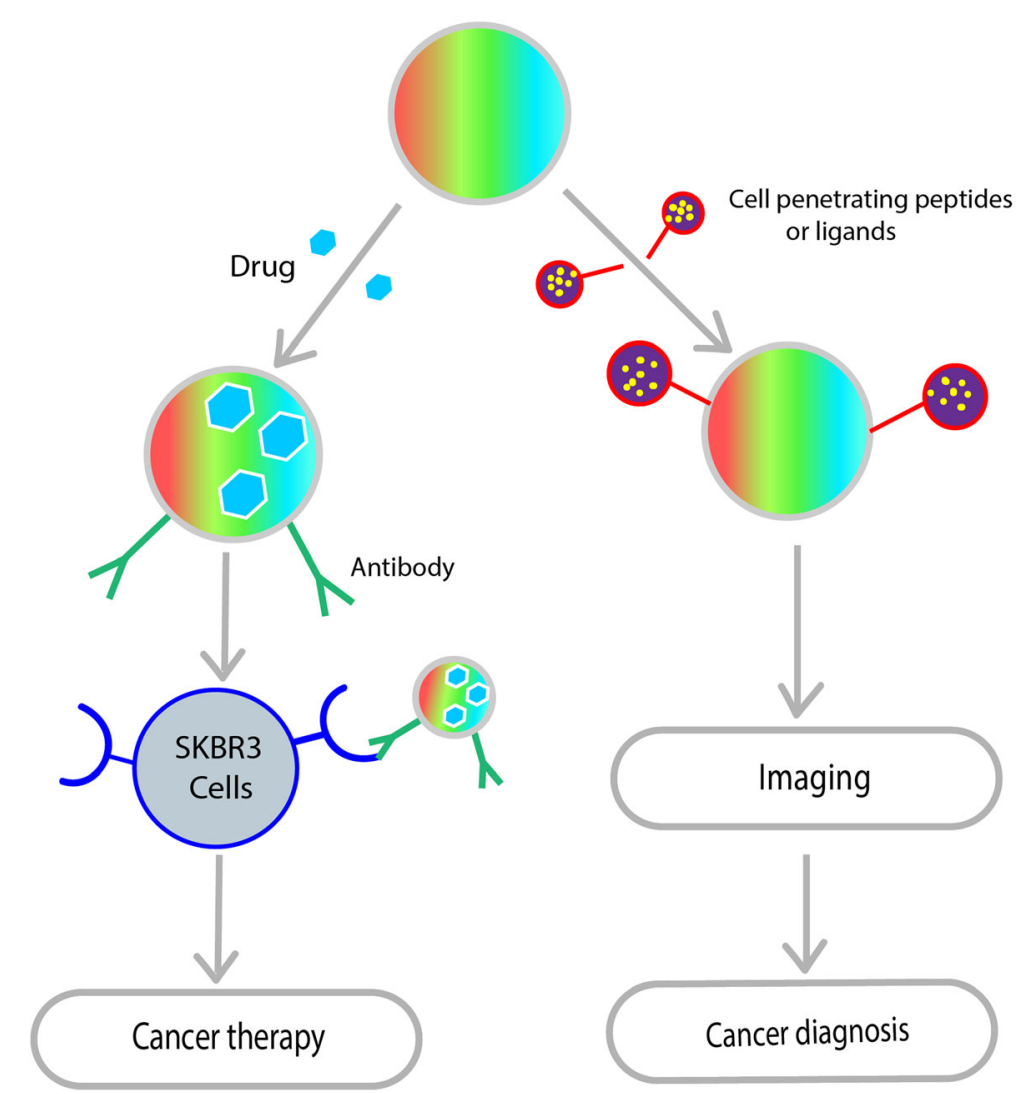

Fig. 6 Ambidextrous nature of QDs in nanomedicine. Theranostics is particularly useful to establish specific or molecular targeting in a single agent (QDs). A range of fluorescent semi-conducting nanocrystals can acts as theranostic agent. Because of its ability to accommodate various functional modalities either targeting agents (antibody, aptamer or protein) or cell-penetrating ligands can be incorporated into QDs for cancer therapy or diagnosis 
direct visualization, monitoring of biomolecular events and physiological process and in vivo deep-tissue imaging [90]. The modification of size and shape of the gold nanoparticles covered with borosilicate glasses have induced greater variations in optical properties [91]. Metallic nanoparticles like Ag nanoparticles (Ag NPs) can exhibit controllable optical properties and the optical property could be enhanced when it is combined with organic solar cells (OSC). Reactive oxygen species or ROS are a group of highly reactive molecules involved in many cellular processes, especially chronic diseases like cancer, diabetes and heart diseases. Additionally, they play an important role in cell signaling pathways; hence, quantification of ROS is highly sought. Traditional optical-based analytical techniques have suffered from low-detection limit fluorescent-gold $(\mathrm{Au})$ nanoparticles having the potential alternative to improve sensitivity. More advanced methods are often introduced to overcome the aforementioned limitations. In this regard, an enzyme immobilized onto graphene-electrode was developed for biosensors applications. A single nanoparticle ( $\mathrm{Au}-$ Pt) based optical sensor was investigated at a nanomolar level for the detection of ROS from microbes and aquatic environments [92].

UV light-responsive drug delivery system (DDS) has its own disadvantages including poor tissue penetration and cell toxicity as a result of UV light exposure and hence is not suitable for clinical practice. Therefore, NIR is considered more promising for clinical application due to spatiotemporal control and considerable penetration [93]. As part of anticancer therapy, multifunctional dimercaptosuccinic acid (DMSA) coated iron oxide $\left(\mathrm{Fe}_{3} \mathrm{O}_{4}\right)$ nanoparticles with doxorubicin (DOX) have demonstrated excellent cell toxicity to human breast cancer (MDA-MB-231) cells via the synergistic effect of $\mathrm{pH}$ and NIR-light induced photothermal therapy combined with chemotherapy [94]. Based on these results, it was stated that the $\mathrm{Fe}_{3} \mathrm{O}_{4} @ \mathrm{DMSA} / \mathrm{DOX}$ nanoparticles may work as an effective anticancer therapy for breast cancer. With suitable surface modification, a multifunctional $\mathrm{Zn}-\mathrm{Fe}_{2} \mathrm{O}_{4}$ nanoparticle was designed as an anticancer drug carrier for the hydrophobic molecule curcumin and the hydrophilic molecule daunorubicin in cancer therapy [95]. The hydrophobic-hydrophobic interaction between curcumin and long-chain surface ligands of $\mathrm{Zn}-\mathrm{Fe}_{2} \mathrm{O}_{4}$ nanoparticles favored the incorporation of the drug molecules into the alkyl chain of oleic acid-coated $\mathrm{Zn}-\mathrm{Fe}_{2} \mathrm{O}_{4}$ nanoparticles. Whereas, the daunorubicin drug molecules were adsorbed on the surface of the nanoparticles via electrostatic interaction. Development of such multifunctional nanoparticles has been found to have promising application in dual drug delivery applications.

\section{Magnetic property}

The concept of magnetic property in drug delivery was introduced in the year 1978 [96]. Considering the technical advancements in MNPs design and in vivo studies, MNP based drug delivery has received much attention in the field of nanomedicine [97]. The route of administration will have a direct impact on poor drug bioavailability, especially if administrated via the systemic route due to incomplete absorption or degradation. In conventional drug delivery (i.e., injection or ingestion of the desired drug), each drug has its own therapeutic range above which it is toxic and below which it is ineffective. This is because the oscillating drug concentration will result in either ineffectiveness or toxicity [98]. To overcome the limitations of conventional drug delivery system, specific target-hitting drug delivery systems are required and anticipated to provide more effective drug accumulation in the diseased site. Even in targeted drug delivery, a significant quantity of injected PEGylated liposomal DOX was seized by lysosomal sequestering after an internalization that resulted in limited efficacy of the drug [99].

A magnetic drug delivery system is generally comprised of an iron oxide nanoparticle with the drug of interest and they are delivered to the tumor site with the aid of external magnetic field [100]. The magnetic property is not only useful in delivering drugs to the target site but also useful in gene delivery, a contrasting agent in MRI and cell separation, etc. Among the various MNPs, iron oxide is the most preferred material as it is biocompatible, biodegradable and most importantly, possesses superparamagnetic (SPM) behavior [101]. The MNPs are often coated with polymers such as dextran, starch, and PEG to stabilize the core iron oxide nanoparticles. Consequently, particle aggregation will be reduced; however, it also decreases the magnetization saturation of bare iron oxide nanoparticles. The SPM behavior of nanoparticles is defined by lack of hysteresis loop, coercivity and remanent magnetization at room temperature [102]. When the nanoparticles are synthesized at the size range of $10 \mathrm{~nm}$ without surface modification, it offers negligible remanence and coercivity in the magnetization curves. The small size and surface effect of the particle will determine the magnetic responsiveness of the material $[103,104]$.

Designing of such nano-scale SPM materials has a significant impact on nanomedicine including magnetic resonance imaging application for neuro-oncology [105], drug delivery via magnetic drug targeting [106] and enhanced hyperthermia by iron oxide nanorods [107]. The dual targeting of drug delivery by magnetic nanoparticles (MNPs) combined with liposomes is another recent trend in cancer therapy [108]. Bubble-generating magnetic liposomal (BML) drug delivery system is triggered 
with drug release properties for targeted delivery of doxorubicin in cancer therapy. BML was obtained by treatment of liposomes with citric acid-coated iron oxide MNPs co-entrapped with ammonium bicarbonate by simple hydration and surface modified with hyaluronic acid-polyethylene glycol (HA-PEG) coating [109]. The resultant liposomes are effective in delivering increased DOX concentration to the human glioblastoma cells (U87) cancer cells through temperature-sensitive drug release thereby improving targeting as well as treatment efficiency.

\section{Particle size}

Nanoparticle applications are predominantly governed by its properties wherein particle size and size distribution are crucial as size will easily influence the drug loading, release, toxicity, in vivo distribution and particle stability, etc. One of the biggest limitations in nanoparticle aided drug delivery is clearance by the reticuloendothelial system (RES) through opsonization and it is implicit here that the size influences clearance as well as distribution. When the particle size exceeds $100 \mathrm{~nm}$, the pharmacokinetic and biodistribution properties greatly change and they are detected in blood and organs like spleen, lungs, liver, and kidney [110]. Positively charged NPs show better uptake by direct permeation than neutral and negatively charged NPs [111]. Nanoparticle size or the particle diameter can be controlled either by the fabrication methods or adjusting the physical properties, particularly concentration of the polymer or the surfactant. For brain targeted drug delivery systems, the difficulty of treating brain tumor is overcome by shrinking endothelial cells and opening endothelium tight junctions for the delivery of chemotherapeutics across the blood-brain barrier (BBB) [112]. To improve the paracellular transport, tight junctions can be opened only to a certain extent and particles of $<20 \mathrm{~nm}$ can penetrate the brain via the $\mathrm{BBB}$. The $\mathrm{BBB}$ disrupting properties of hyper-osmotic mannitol facilitate effective penetration of nanoparticles across the BBB [113]. For such effective penetration, the particle diameter should ideally be 10 $150 \mathrm{~nm}$ as it will sustain longer circulation time and increased accumulation in the target site [114]. The rate of drug release can be tuned by particle size and, in case of large particles, more drug molecules can be accommodated and slowly released [115]. Although the smaller nanoparticle has a high surface-volume ratio, they can easily be aggregated and may be released quickly since they adhere to the edge of the particle surface.

\section{Morphology}

It is clear that the number of nanoparticle properties i.e., particle size, charge and surface have a great effect on drug delivery. Besides, nanoparticle shape has also been significantly useful in the development of nanocarriers (NC). The significance of nanoparticle shape in drug delivery has been discussed by several authors $[116,117]$. However, the precise role of particle shape in drug delivery has yet to be delineated. The shape of the nanoparticle is always dependent on the synthesizing methods where methods like ab initio are used to produce particles with non-spherical geometry [118]. Since the nonspherical particles may have two or more different lengths, one length could dominate the other. Irrespective of the different administration routes, particle shape will greatly affect the transport and diffusion of nanoparticles. It has been shown that the sphere-shaped particles move easily due to their inherent symmetry whereas the non-spherical ones tumble with the flow. This will be more prominent in filtering organs like spleen and liver. Folic acid-targeting folate ligands in the form of spherical and wormlike micelles (75 and $200 \mathrm{~nm}$ ) using acrylic acid (AA) and PEG methyl-ether acrylate (PEGMEA) were intended for drug delivery [119]. When compared to spherical particles, wormlike micelles were highly accumulated in the spleen, liver, and kidneys. Long filomicelles should be stretched out whenever vflow $>5 \mu \mathrm{m} \mathrm{s}^{-1}$, which includes flow in most blood vessels and also the filtering spleen [120]. It was reported that the shape, geometry, and orientation of the particle would greatly influence the cellular uptake $[121,122]$ and, even in cases of non-spherical particles larger than $200 \mathrm{~nm}$, can still pass through the spleen provided one of their dimensions is less than $200 \mathrm{~nm}$ [123]. The targetspecificity of nanoparticles is also subjected to the shape of the nanoparticle which may eventually result in longevity and internalization of particles. Therefore, it was concluded that the symmetry of nanoparticle is crucial for effective drug delivery.

\section{Surface tailoring}

The wide-spread clinical applications of nanoparticles fostered studying the interaction between the nanoparticle surface and the inner biological system, especially, at physiological conditions ( $\mathrm{pH}$ of 7.0 to 7.4). Based on the choice of application, a nanoparticle with desired property is selected, e.g., optical-gold NPs [90], magnetic-iron oxide nanoparticle (IONPs) [124, 125], fluorescencequantum dot, etc. [126]. Before introducing such a nanoparticle into the environment, it needs to be carefully modified with the appropriate functional groups by a suitable fabrication method. The mentioned surface engineering approaches not only offer an excellent stabilization in an aquatic system but also effectively deliver the drug to the target site. The particle stabilization is often achieved through ligand immobilization or polymer coating. Binding of a ligand on the surface of a nanoparticle would prevent agglomeration by a repulsive force, which results in 
the control of nanoparticle size and shape [127]. When the nanocarriers are introduced into the biological system, the proteins in the biological fluids will commence being adsorbed into the nano surface and form a protein-rich layer (protein corona). The resultant protein-corona and nanoparticle complex have protective effects on the biological system; however, the molecular complexity of protein-corona nanocarrier is still not well investigated [128]. Formation of protein-corona will occupy the surface of nanoparticles and block the chemical functionality as offered by the nanoparticle. Besides, it will have effects on particle size and size distribution, which directly influences the circulation time, intracellular trafficking and clearance/cell uptake process [129]. Likewise, nanoparticle surface chemistry plays a key role in the cellular uptake process. Polymer coating of the NP surface has considerably reduced the chance of particle clearance by the immune system and avoided accumulation in other organs [130]. The benefits of the polymer coating (e.g. PEG) is to control protein or peptide absorption via its hydrophilic chains that will also regulate cell behavior during contact. Desirable functionality can be added to the particle by methods utilizing monotopic capping agents. However, fabricating this the right way still remains a challenge [131].

\section{Drug-laden nanocarriers}

The name "nanocarrier" suggests that the materials belonging to this category are $1-100 \mathrm{~nm}$, but size ranges > $200 \mathrm{~nm}$ are generally to be avoided because particle size has a significant effect on circulation time. This is especially true with smallest capillary dimension as the possibility of obstruction exists. Drug-laden nanocarriers are prepared by various synthesis methods and one of the best-suited methods is nano-encapsulation [132]. Emulsion polymerization is a method wherein natural or synthetic polymers are subjected to a continuous aqueous or organic phase. The selection of nanoparticle-drug formulation is decided by the physicochemical properties of the drug viz. drug solubility nature, chemical stability, etc. In the continuous organic phase methodology, polymers are added along with the surfactants to prevent aggregation. The method also exploits initiators and toxic organic solvents for preparation. Hence greater emphasis is placed on alternate methods with more safety. In the continuous aqueous phase, mostly antibiotic or drug molecules are encapsulated in the nanoparticle using aqueous solution without surfactant or emulsifiers. The synthesis of poly (methyl methacrylate) (PMMA) nanoparticle to carry influenza viral adjuvant is a classic example of continuous aqueous phase polymerization produced through radical emulsion polymerization [133]. In addition to the antigen example, various drugs like doxorubicin, ketoprofen, and insulin were also nano-encapsulated [134].

Some of the nanoparticle formulations have offered improved and higher oral availability of low-water soluble drugs. Most of the anticancer drugs (paclitaxel, docetaxel), small molecule anticancer drugs [VEGFR inhibitors (e.g. cabozantinib, nintedanib] and compounds like curcumin have exhibited poor solubility and, even today, the solubility range of recently developed anticancer compounds are at the $\mu \mathrm{g} / \mathrm{mL}$ range [135-137]. The feasibility of using a nanocarrier is not restricted to improving the bioavailability. It also has additional benefits: reduced systemic toxicity, enhanced tumor accumulation and improved therapeutic effectiveness by selective drug aggregation [138]. Among the different types of nanocarrier systems, nano-formulations based on lipid, polymer, and albumin are widely studied for its encapsulation and delivery of the existing as well as new chemotherapeutic drugs. Pyrazolo[3,4-d] pyrimidines demonstrated promising anticancer activity against many different cell lines but like many other anticancer compounds, it displayed poor aqueous solubility. This potential limitation was overcome by encapsulating in nanosystems like albumin nanoparticles and liposome and in the end, it showed remarkable pharmacokinetic profile [139].

Although therapeutic proteins are approved by the FDA for various disease-designated purposes, the main drawback of therapeutic proteins is that low half-life and lack of stability. Liu et al prepared interferon conjugated with an alpha block copolymer to form IFN-POEGMAPHPMA [poly (oligo (ethylene glycol) methyl ether methacrylate)-poly(2-hydroxypropyl methacrylate)] micelle and compared its tumor activity against the US FDA approved IFN- $\alpha$ PEGASYS (Peginterferon $\alpha-2 a$ ). The results showed complete suppression of tumor in mice model when administered with IFN-micelle. While PEGASYS and IFN-POEGMA were effective, IFN at the same dose $(1 \mathrm{mg} / \mathrm{kg})$ was not as efficacious. The in vitro bioactivity of the micelle was 21.5 fold higher than that of the FDA approved interferon. The result indicates that stability and therapeutic efficiency can be increased by conjugating with polymer [140].

\section{Factors influencing the biodistribution of drug-laden nanocarriers}

It is believed that numerous factors could affect drug loading capacity besides synthesis methods and reaction conditions. Chitosan-grafted-glycerides (monooleate, monolaurate, and monostearate) were synthesized to achieve a successful transport of drugs across the complex intestinal barrier. This study also reported that the selection of optimum copolymer and drug is equally essential in the preparation of a stable micelle system and it was achieved using computational simulation [141]. Each nanoparticle or nanocarrier 
system has a distinct chemical composition and size variation. If the carrier is not surface modified with suitable agents, it is rapidly cleared from the bloodstream by mononuclear phagocyte system (MPS) (this process is called phagocytosis), the liver or the spleen [142]. Hence, an important aspect of designing nanocarrier is fabricating the nanosystem with optimal clearance characteristics with particle material, size, shape, surface chemistry, and charge being some of the properties that would influence this clearance. Ideally, the size would be bigger than blood capillaries to avoid leakage yet tiny enough to hide away from macrophage engulfment. To overcome the numerous biological barriers, surface modified carriers are increasingly described for targeted drug delivery and it could be achieved by incorporating desired functionality or characteristics on the nanoparticle by suitable synthesis methods. The surfacemodified carriers are expected to provide prolonged circulation time and minimize the risk of opsonization. P-glycoprotein (P-gp) is an efflux membrane transporter found to be overexpressed in cancer cells and act as a physiological barrier. It obstructs chemotherapeutic agents from entering the cytosol by extruding them to the exterior during anti-tumor treatments [143]. Polysorbate 80 has been demonstrated as an inhibitor of P-gp and its potential P-gp inhibition results in the delivery of a significant amount of doxorubicin using nanoparticles with polysorbate 80 coating [144].

\section{Targeted drug delivery methods}

\section{Passive targeting}

Drug targeting is defined as the selective drug release at a specific physiological destination organ or tissue or cell in which specific pharmacological impact is required. Nanocarrier mediated cell targeting includes active and passive mechanisms. In passive targeting, the drugs can be delivered to the target organ passively based on the longevity of the pharmaceutical carrier in the blood and preferential accumulation of the drug-loaded nano delivery system at the site of interest [145]. The main property of tumor tissues is that they have defective blood vessels and hence exhibit increased vascular permeability. This unique characteristic helps to transport macromolecules into tumor tissues. Maeda et al have demonstrated that the site of infection or inflammation where excess bradykinin is generated also exhibits enhanced permeability and retention (EPR) effect [146]. The main difference between the infection-induced EPR effect and that of tumor is the duration of the retention period. In the case of normal tissues, the time will be shorter due to swelling while in cancer tissues the lymphatic drainage system is active. Thus swelling may disperse after a few days. In cancer, the enhanced vascular permeability results in adequate nutrients and oxygen supply to the tumor tissues for their rapid growth. This unique anatomical-pathophysiological nature of tumor blood vessels is being exploited to deliver drug molecules to the tumor tissues. Macromolecules bigger than $40 \mathrm{kDa}$ will spill out from the tumor vessel and concentrate in tumor tissues. Normal tissues lack this EPR effect driven drug delivery. This unique EPR effect feature of tumor cells is subsequently thought to be a milestone principle in tumor-targeting chemotherapy and is turning into an inexorably encouraging worldview approach for anticancer drug development. Hence, it has become the golden standard in anticancer drug design and anticancer strategies using macromolecular agents, including gene delivery, molecular imaging, antibody therapy, micelles, liposomes, and protein-polymer conjugates [147-149]. PEG is the most important polymer used to modify proteins to enhance the efficiency of drug delivery. PEGylated Lasparaginase has a circulation lifetime of 5.7 days in humans compared to 1.2 days for the original enzyme [6] and was successfully used as induction therapy for phase-3 acute lymphoblastic leukemia (ALL) [150]. Several proteins-polymer conjugates are already available as anticancer agents. In some cases, blood plasma components are capable of increasing circulation time. A study by Gradishar et al revealed higher response when nanometersized albumin-bound paclitaxel (ABI007) was administered intravenously in women with metastatic breast cancer than standard paclitaxel formulation [151]. Similarly, the ABI007 nano-drug showed a 4.5 -fold increase in paclitaxel transport across endothelial cells compared to standard paclitaxel [152]. Taxol ${ }^{\circ}$, when loaded into micelles made of PEG- $\beta$-poly(4-phenyl-1-butanoate)-1 aspartamide conjugate, showed almost a 100-fold increase in the area under the curve (AUC), a 15-fold decrease in the volume of distribution and a significant decrease of drug clearance was achieved resulting in a 25-fold improvement in drug accumulation in C-26 tumors in mice [6]. Polymer-conjugates styrene-maleic anhydride-neocarzinostatin (SMANCS), the PEG-granulo cyte colony-stimulating factor and PEG-L-asparaginase are currently available in the market and are being used against hepatocellular carcinoma, acute lymphoblastic leukemia and chemotherapy-associated neutropenia, respectively [153]. Passive targeting cannot deliver large solutes and there arises the need for alternative tactics which has led to the development of active methods (Fig. 7a) [155].

\section{Active targeting}

Active targeting is based on the attachment of a specific site to the surface of pharmaceutical carriers. It makes use of molecular recognition patterns like ligandreceptor, antigen-antibody to deliver drugs to a specific location (Fig. 7b). This strong interaction confers more specificity to the delivery system. The active strategy can 


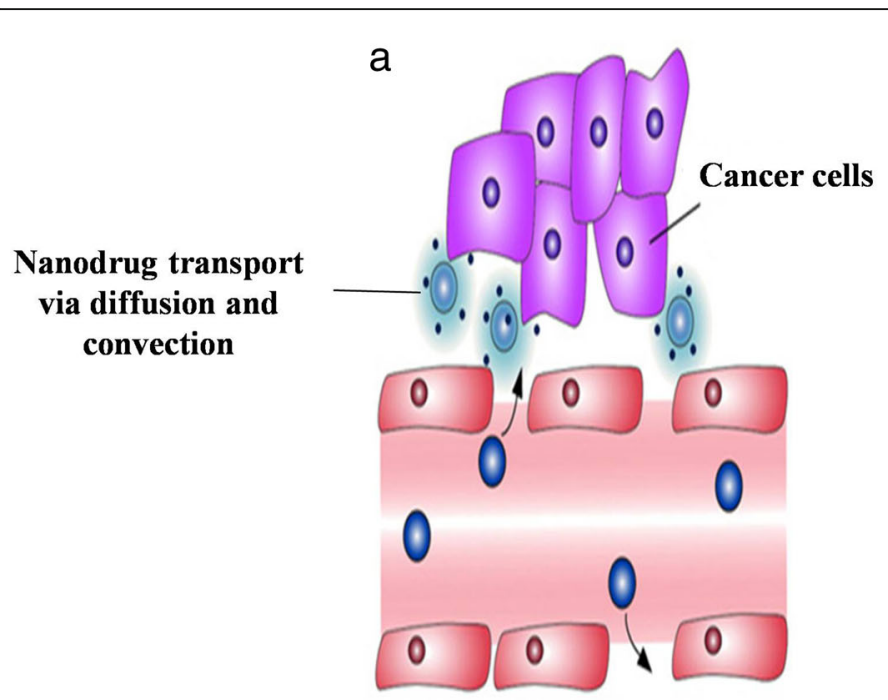

Fenestrated vasculature

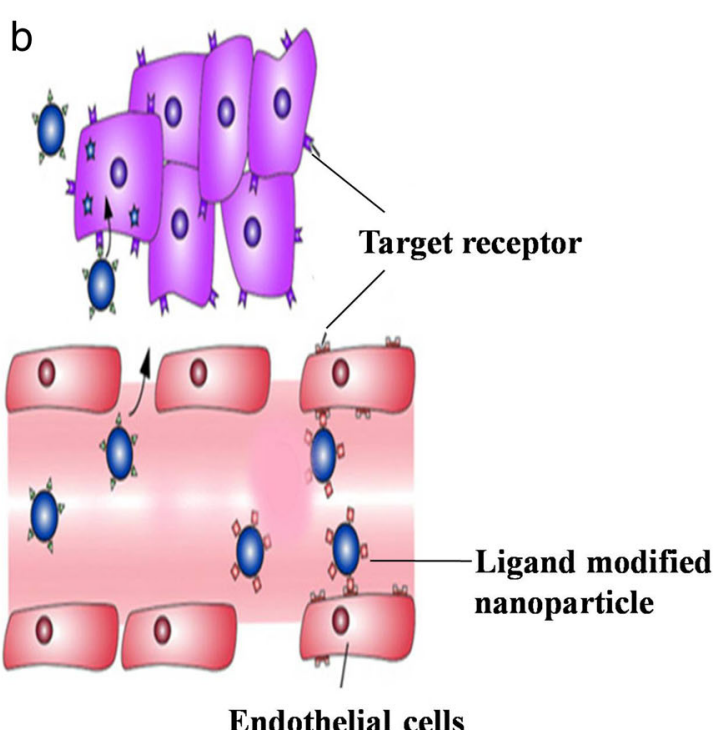

Endothelial cells

Fig. 7 Drug delivery through passive and active targeting. Enhanced vascular permeability is one such hallmark feature of tumor cells along with the defective vascular anatomy. a Passive targeting uses this feature and improves the drug delivery by convection or passive diffusion in tumor cells. $\mathbf{b}$ Whereas in active targeting, targeting ligands are over expressed in tumor cells, thus the coveted nanoparticles are engineered to incorporate ligand that will bind to the target cells through ligand receptor interaction. This in turn increase the efficiency of drug delivery to the tumor tissues [Adapted from reference with permission: Wicki A, Witzigmann D, Balasubramanian V, Huwyler J Nanomedicine in cancer therapy: challenges, opportunities, and clinical applications. J Control Release 2015; 200:138-157] [154]

be also achieved through the manipulation of physical stimuli (e.g., temperature, pH, magnetism) [138]. In active targeting, the ligand is coupled onto the nanoparticle surface that will interact with its receptor in the target site. The success of drug targeting relies upon the choice of targeting moiety which ought to be abundant, have strong affinity and specificity to bind cell receptors as well as be suitable to chemical modification by conjugation. The active targeting ligands for tumor therapy include folate, transferrin, aptamers, short oligonucleotides of RNA or DNA that can fold into various conformations and engage in ligand binding, antibodies, and peptides, etc. Active targeting offers less toxicity to healthy tissues as targeting ligands are overexpressed on the tumor tissue, so it is widely used for cancer treatment [99, 114, 156]. Poor tumor targetability and multidrug resistance (MDR) are two major impediments to the success of cancer treatments. In the case of specific-drug targeting, internalization of nanoparticles over receptor-mediated cell interactions are considered an effective method. A large number of epithelial cancers have the characteristic overexpression of folate-receptors; hence, they are targeted for effective chemotherapy [157]. Ethoxy-(poly(ethylene glycol))-folic acid (FA-PEG) micelle consist of docetaxel (DTX) used to exert higher toxicity on FR-positive MCF-7 cells [158]. Hyaluronic acid (HA) or its derivatives are increasingly used to target and bind to overexpressed cell-surface receptors on the tumor cells and can deliver various anti-tumor drugs, proteins and nucleic acids [159].
HA-paclitaxel conjugate (HA-PTX) has shown superior anti-tumor activity against head and neck squamous cell carcinoma cell lines OSC-19 and HN5 upon binding to $\mathrm{CD} 44$ receptor, increasing the uptake of the polymer-drug conjugate [160].

Fabrication of matrix metalloproteinases (MMP)-responsive smart drug delivery system is a new way to inhibit MMPs expression as MMPs are widely considered cancer biomarkers. Such targeting systems are developed by incorporating the MMP substrates (collagen, gelatin, fibrinogen, etc.) into nanoparticles. However, large proteins have serious limitations in drug targeting or delivery. The synthetic MMP substrates (i.e., MMP-sensitive peptides) are not only easy to incorporate but also offer selectivity and sensitivity. Yet the MMP responsiveness of the nanoparticles varies with the choice of peptides used [161]. A new type of self-assembling polyethylene glycol-phosphoetha nolamine-based copolymers (PEG-pp-PE) was designed for treating drug-resistant cancers by inhibiting both the matrix metalloproteinase 2 (MMP2)-sensitive tumortargeting and P-glycoprotein (P-gp)-mediated drug efflux [162]. The molecule size and surface attributes of nanoparticles can be effectively controlled to accomplish both passive and active drug targeting with fewer side effects. Nanoparticle addition shields the drug from degradation. This system can be utilized for different routes of administration including oral, nasal, parenteral, etc. The drug will remain at a specific site in the right proportion for a prolonged time with less wastage and efficacy $[163,164]$. 


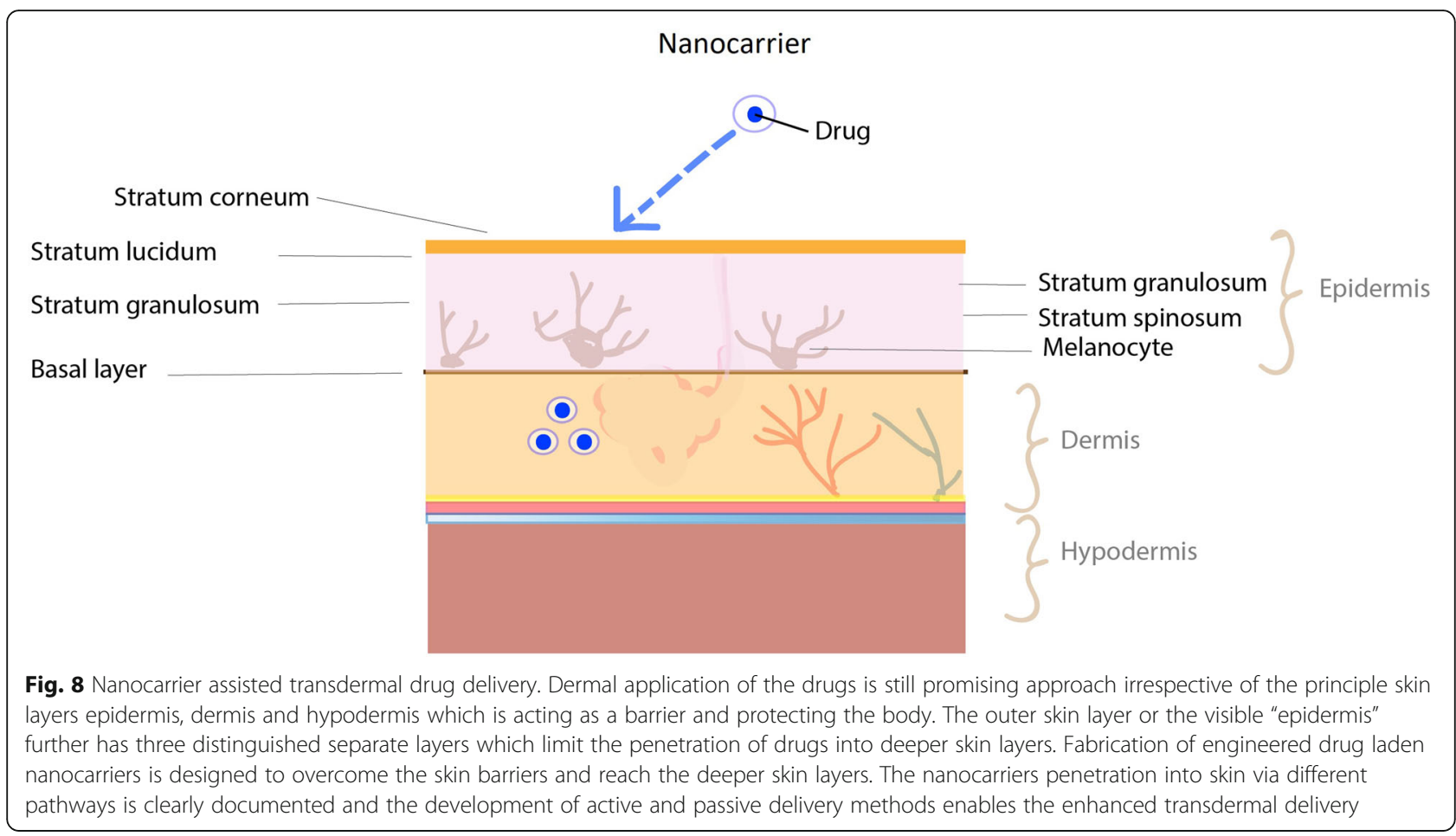

\section{Different administration routes of Nanocarriers}

\section{Transdermal drug delivery (TDD)}

Human skin is the largest organ in our body covering a surface area of $1.8-2.0 \mathrm{~m}^{2}$. It is composed of three main layers: the epidermis, dermis, and hypodermis (Fig. 8). The outermost epidermis layer is made up of $95 \%$ keratinocytes and the remaining percentage consists of Langerhans cells, melanocytes, and Merkel cells. The outermost layer of the epidermis, stratum corneum consists of anucleated physically dead keratinocytes called corneocytes presenting a thickness of $10-20 \mu \mathrm{m} \quad[165,166]$. The multilayered brick and mortar structure of keratinocytes, together with their lipophilic nature of the stratum corneum is responsible for the barrier property of the skin [167]. The primary goal of a nanocarrier is to overcome the stratum corneum barrier. NCs such as nanoemulsions, vesicular (liposomes, ethosomes, niosomes, etc.) and nanoparticular NCs are developed to overcome this obstruction [168]. Nanoparticles enter the skin through (1) the intercellular pathway (lipid matrix occupying the intercellular spaces of the keratinocytes), (2) the transcellular pathway (through keratinocytes) and (3) the transappendageal pathway (across hair follicles, sebaceous glands, and sweat glands) [169].

Since skin appendages cover only $0.1 \%$ of the skin surface area, initially it was considered as a non-important route for drug penetration. Nevertheless studies have shown that hair follicles could be an interesting option for drug penetration through the skin [34]. Lademann et al investigated the storage behavior of the dye containing nanoparticle $(320 \mathrm{~nm})$ and non-particle form on human skin and found that the nanoparticle formulation stored in hair follicles up to 10 days, while non-particle form could be detected up to 4 days [170]. The surface images of topical administrated polystyrene nanoparticle on porcine skin (ex vivo) and human skin (in vivo) have revealed NPs accumulation in the follicular openings. The CLSM (confocal laser scanning microscopy) images showed accumulation of F-NP $(20 \mathrm{~nm})$ were almost the same in hair follicles and skin appendages after $30 \mathrm{mi}-$ nutes. Increasing exposure time for about 1 to 2 hours displayed a better accumulation in hair follicles than skin appendages. The results conveyed the time dependent distribution of naoparticle accumulation in hair follicles [171]. The TDD system prevents the first pass metabolism effect of drugs. Therefore, lower amount of drug can be administered efficiently with reduced toxicity. The main disadvantages of TDD system are that not all drugs can be delivered transdermally. High molecular weight drugs $(>500 \mathrm{Da})$ are not capable of penetrating the stratum corneum [34, 172].

\section{Nanocarriers for transdermal drug delivery}

The commonly used nanocarriers for dermal/transdermal drug delivery in the pharmaceutical field include liposomes, transfersomes, ethosomes, niosomes, dendrimers, polymer nanoparticles, and nanoemulsions. Liposomes are closed colloidal carriers composed of phospholipids and steroids. They can carry hydrophilic drugs inside their core and lipophilic drugs between the lipid bilayer. Liposomes may be negatively or positively charged and their deformability 
decreases by increasing the amount of cholesterol in their composition [173]. In 1995, the US FDA approved the first liposome-encapsulated drug Doxil (PEGylated liposomeencapsulated doxorubicin) for the treatment of AIDSrelated Kaposi's sarcoma, later approved for ovarian cancer. Recently, it has also been approved for the treatment of breast cancer in the USA and the treatment of multiple myeloma in combination with Velcade, a proteasome inhibitor, in Europe and Canada [174-176].

To improve skin permeation and increase efficiency, the composition of liposomes are altered to the newly generated classes of lipid vesicles called transferosomes, niosomes, ethosomes, etc. Transfersomes are negatively charged elastic or deformable vesicles composed of phospholipids as their main ingredient with 10 to $25 \%$ surfactant (such as sodium cholate) and 3 to $10 \%$ ethanol. The presence of surfactants destabilize the lipid bilayers of vesicles and confer their ultradeformability thereby enabling them to squeeze themselves through the narrow pores in the stratum corneum that are less than one-tenth the diameter of the transfersome. While liposomes cannot penetrate the channels, transferosomes, up to $500 \mathrm{~nm}$ in size, can penetrate the stratum corneum [177]. Niosomes are vesicular nanocarriers formed by the assembly of non-ionic surfactant in an aqueous phase. Niosomes are developed to deliver both lipophilic and hydrophilic drugs [178]. The unique property of niosomes is that they reduce the systemic absorption of drug which in turn enhances the residence time of the drugs in stratum corneum. The role of surfactant is to enhance the penetration of the drug by adsorption at the interfaces or by interacting with biological membranes and by modifying the barrier function of the stratum corneum. Examples of transdermal drug delivered using niosomes are Minoxidil, an antihypertensive vasodilator medication, and ellagic acid (EA) [34, 179]. Pomegranate ellagic acid (PEA) is a natural polyphenol that possesses excellent antioxidant, anti-tumor, anti-inflammatory, antibacterial, and skin whitening properties. However, the characteristics of low permeability and poor absorption rate of EA have limited its application. The pomegranate ellagic acid-hydroxypropyl- $\beta$-cyclodextrin (PEA-HP- $\beta$-CD) inclusion complex was prepared to offer an enhanced drug effect via effective transdermal permeation [180]. Similarly, EAloaded niosomes were also used as an effective carrier for the dermal delivery of EA [181]. Ethosomes consists of the stratum corneum. Ethosomes consist of phospholipid/surfactant, water and ethanol ( 30\%). Drugs encapsulated in ethosomes can penetrate deep skin. The presence of a high amounts of ethanol helps in breaking the stratum corneum [182]. Tacrolimus, Clotrimazole, Trihexyphenidyl $\mathrm{HCl}$, Ketoprofen and testosterone have been delivered using ethosomes [34]. Nanoemulsions are a dispersion of oil and water stabilized by an emulsifying agent. Their size varies from 100 to $1000 \mathrm{~nm}$. They are transparent due to the droplet size being less than $25 \%$ of the wavelength of visible light $[183,184]$.

\section{Methods involved in transdermal drug delivery}

Passive methods can deliver only a limited amount of drug that is of low molecular weight $(<500 \mathrm{Da})$. Active methods have been developed in order to overcome this. It uses mechanical and physical methods to enhance skin permeability. The main advantage of the active strategy is that it can deliver large molecular weight molecules (>500 Da) efficiently. The novel transdermal delivery system focuses on how to overcome the stratum corneum barrier by using microneedles, thermal ablation, microdermabrasion, high pressure-jets, iontophoresis, laser, electroporation, and ultrasound [174]. Most of these methods are currently progressing to deliver macromolecules (heparin, oligosaccharides) and vaccines. Smallpox vaccine administered by microneedle mediated skin electroporation in mice showed a strong immunological response against the pox virus immunogens of interest than traditional live virus administration [185]. A simple inexpensive microporation has been developed to increase the permeability of the skin for the delivery of genetic vaccine using replication-defective adenoviruses (rdAds) [186]. The resultant skin immunization through microporation is not only painless but also enhances the activity of rdAds by up to 100 -fold as compared to intact skin. The advent of these novel strategies has had a great impact on medicine. By addressing the safety, efficacy, portability, user-friendliness, and cost-effectiveness, these novel drug delivery techniques can compete with those already on the market [155, 187-189].

\section{Blood brain barrier drug delivery}

One of the tightest endothelium, the Blood-Brain Barrier $(\mathrm{BBB})$, is present in the central nervous system. The term "blood-brain barrier" ("Blut-Hirn-Schranke") was coined by Lewandowsky in 1900. The human brain contains about 100 billion capillaries with the brain capillary endothelium spread across approximately $650 \mathrm{~km}$ that covers a total surface area of approximately $20 \mathrm{~m}^{2}$. Endothelial cell, astrocyte, pericyte, microglial cells, and adjacent neurons constitute the BBB [190]. The entry of molecules into the brain is regulated by $\mathrm{BBB}$ and bloodcerebrospinal fluid barrier. The $\mathrm{BBB}$ is considered the bottleneck for successful development of the central nervous system (CNS) acting drugs. Most of the neurotherapeutic compounds never reach the market due to their inability to cross the BBB [191].The high selectivity of the $\mathrm{BBB}$ is due to the presence of cerebral endothelial cells. Adherens junctions (AJs) and tight junctions (TJs) present between endothelial cell acts as the physical 


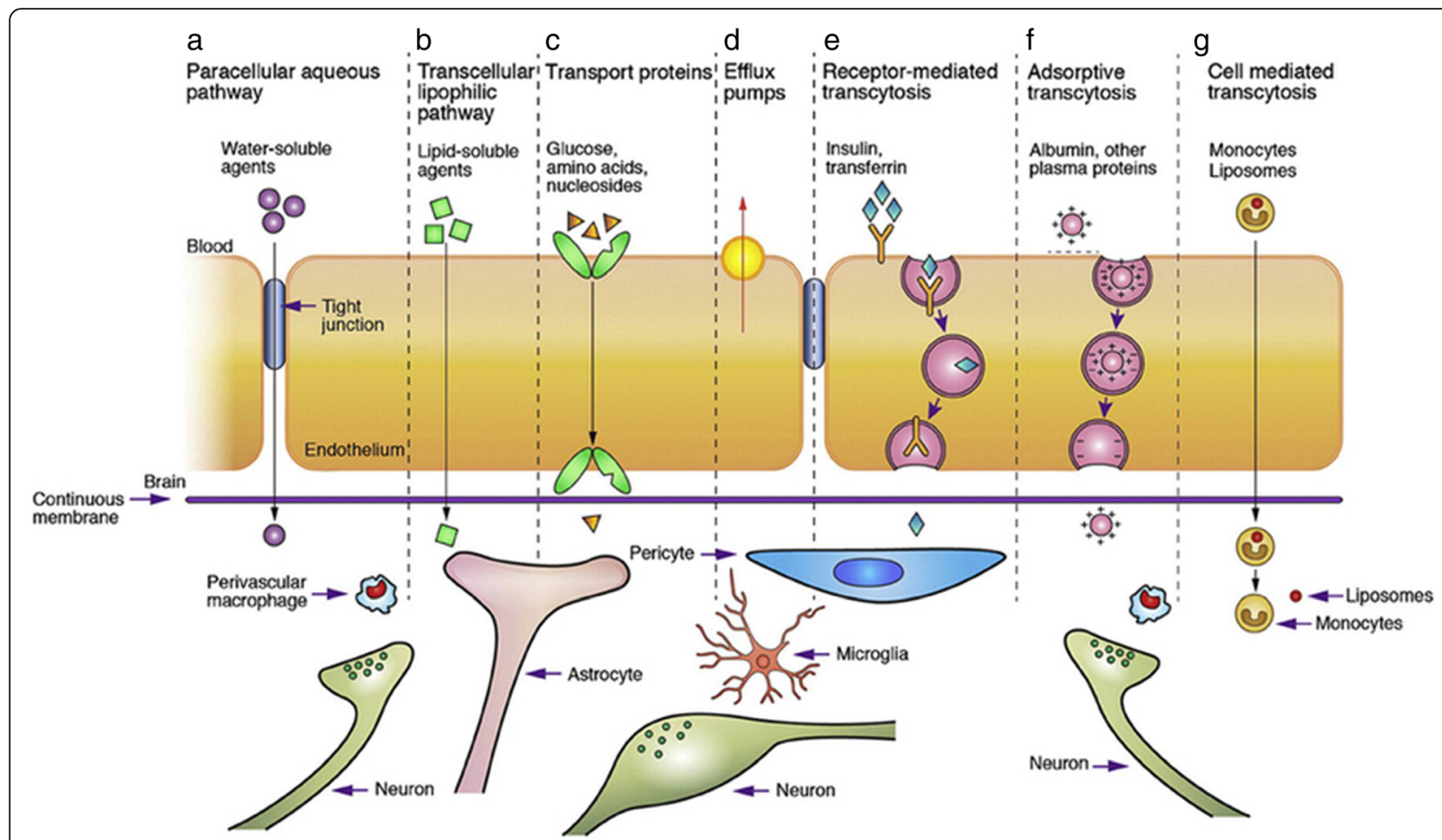

Fig. 9 Transport mechanism through blood brain barrier. Transport routes across the blood-brain barrier. Pathways (a-f) are commonly for solute molecules; and the route $(\mathbf{g})$ involves monocytes, macrophages and other immune cells and can be used for any drugs or drugs incorporated liposomes or nanoparticles. [Adapted from reference with permission; N.J. Abbott, L. Ronnback, E. Hansson, Astrocyte-endothelial interactions at the blood-brain barrier, Nat Rev. Neurosci 7 (2006) 41-53] [192]

barrier. This compact network of interconnections supplies transelectrical resistance $>1500 \Omega \mathrm{cm}^{2}$ to BBB. Microglia, perivascular macrophages, and mast cell serve as the immunological barrier. The transport barrier includes para- and transcellular routes. The transcellular route includes carrier-mediated transport, receptormediated transcytosis, adsorptive mediated transcytosis and cell-mediated transport (Fig. 9) [192, 193]. The intra and extracellular enzymes present in the endothelial cells work as a metabolic barrier against lipophilic substances [194].

\section{Formulation of nanocarriers and its mechanism of delivery}

Several nanocarriers including liposomes and solid lipid nanoparticles have been reported to deliver drugs across the BBB successfully. However, hexapeptide dalargin (Tyr-D-Ala-Gly-Phe-Leu-Arg), was the first drug delivered to the brain coated with polysorbate 80 nanoparticle [195]. The leucine-enkephalinanaloguedalargin was investigated as a model drug to study analgesic effects as well as its penetration across the BBB. Here, the dalargin bound to nanoparticles without polysorbate 80 demonstrated no analgesic effect. The most challenging research has focused on targeted drug delivery across the $\mathrm{BBB}$ to diagnose and treat various neurological disorders. Generally, the transport mechanism across the BBB can be categorized into three mechanisms: receptor-mediated, carrier-mediated, and vesicular transport [196]. Most nanosized systems use adsorptivemediated transcytosis and receptor-mediated transcytosis as the two major mechanisms to deliver neurotherapeutics [197]. The negatively charged cerebral endothelial cells can be made to interact with nanoparticles by adding positive charges. This can be achieved by different procedures. The first method is to make a nanoparticle which bears positive charges at physiological $\mathrm{pH}$ 7.4. The second method makes use of surface functionalization of the nanoparticle with positively charged molecules thereby combining physicochemical features and biological activity. Cellpenetrating peptides like TAT-peptides (derived from HIV) and cationic proteins like albumin are widely used for nanoparticle anchoring that brings about the passage of drugs across the BBB [198].

Receptors for the uptake of different types of ligands (growth factors, enzymes and plasma proteins) are present in endothelial cells. For example, insulin molecules bind to its receptors present in specialized areas of the plasma membrane called coated pits. These coated pits invaginate into the cytoplasm, get separated from 
the cytoplasmic membrane and form coated vesicles. The ligand will dissociate from the receptor after acidification of the endosome [191]. The BBB expresses a variety of receptors including transferrin receptor (TfR), insulin receptor (IR), low density lipoprotein (LDL) receptor, diphtheria toxin receptor, nicotinic acetylcholine receptor (nAChR), scavenger receptors and class $B$ type receptors. Hence, ligands can be used to decorate the deliver system that enables and enhances transport [199]. Qiao et al have successfully developed a brain delivery probe by covalently conjugating lactoferrin to the PEG-coated $\mathrm{Fe}_{3} \mathrm{O}_{4}$ nanoparticles to achieve receptormediated delivery of nanoparticles across the BBB [196]. In carrier mediated transcytosis, carriers mediate the transport mechanism. Carriers for glucose, amino acids, purine bases, nucleosides, and choline are present in endothelial cells and act as a transport system that can deliver the drug. Their main role is supplying nutrients to the brain. In addition, they serve as a transport carrier to deliver drugs. Liposome incorporated mannose derivatives were able to cross the $\mathrm{BBB}$ via a glucose transporter in mouse brain [200, 201].

\section{Methods of $B B B$ drug delivery}

Approaches for delivery of drugs across the BBB can be broadly divided into the following categories: direct injection and implantation, chemical modifications, the temporary opening of the BBB using permeability enhancers and nano-enabled delivery platforms via the intravenous (IV) route and intranasal pathway. Several chemical agents circulating in the plasma membrane or secreted from cells can enhance BBB permeability. Some of the agents that weaken the BBB function include bradykinin, histamine, serotonin, glutamate, purine nucleotides, adenosine, platelet-activating factor (PAF), phospholipase A2 (PLA2), arachidonic acid, prostaglandins, leukotrienes, interleukins(IL-1 $\alpha$, IL-1 $\beta$, IL-6), tumor necrosis factor- $\alpha$ (TNF- $\alpha$ ), macrophageinhibitory proteins MIP1 and MIP2, complementderived polypeptide C3a-desArg, free radicals and nitric oxide, to name a few $[192,202]$.

The drug penetration of the $\mathrm{BBB}$ can be improved by special chemical modifications like lipidization and prodrug approach. Chemical modifications focus on the structural rearrangement of the drug to enhance their physicochemical properties. In lipidization, lipid molecules are added at the polar end of the drug molecule providing better permeability than the normal drug [202]. In the pro-drug approach, the drug is distinctively modified to enhance the capillary permeability. For that, the pro-moiety has to permeate through the membrane and once it reaches the brain, the conversion of prodrug to the active parent drug will take place by enzyme catalysis [203]. Neural therapeutic agents can be delivered quickly within minutes by nasal administration. Lower molecular weight drugs having higher lipophilicity can easily enter the central nervous system. These drugs pass through the olfactory nerve where they first enter the respiratory epithelium followed by entry into the systemic circulation. This method delivers drugs into the deeper regions of the brain. The main limitation of this delivery system is that only small molecular weight drugs can be delivered efficiently. Some drugs cannot be internalized by the olfactory sensory neurons in the olfactory epithelium and hence cleared from the CNS easily. The nanoparticle drug delivery system overcomes this limitation and improves the persistence of the drug in the CNS. For example, chitosan modified molecules showed much longer residency time on the olfactory epithelium [204]. PEG-PLGA nanoparticle coated with odorranalectin that has low immunogenicity is widely used as a carrier for the nose to brain delivery. Lactoferrin conjugated PEG-PLGA nanoparticles and poly(ethylene glycol)-poly( $\varepsilon$-caprolactone) polymersomes conjugated with mouse anti-rat monoclonal antibody OX26 are also commonly used [200]. Due to mucociliary mechanisms the drugs get easily removed from the delivery site, reducing the contact period with nasal epithelium and delivery into the CNS following intranasal administration. To enhance the brain uptake and effective drug delivery, mucoadhesive in combination with microemulsion are used. To increase brain uptake and escape from clearance by P-gp mediated efflux, intranasal pretreatment with an inhibitor such as rifampin before intranasal administration of a P-gp substrate like verapamil is recommended [202, 205].

\section{Oral-route of administration}

Oral delivery is the most common route of drug administration with high levels of patient acceptance. The oral route is the most preferred route for drug administration due to greater convenience, pain avoidance, efficacy, high patient compliance, and risk reduction of crossinfection and needle stick injuries [206]. It is expected to overcome the disadvantages associated with injection such as tissue injury, pain, adverse reactions, and poor patient compliance. However, the oral availability of the drug depends on the solubility and permeability of the compound [207]. Furthermore, oral delivery of peptides or proteins frequently suffers from the acidic environment and enzymatic system of the gastrointestinal tract (GIT) leading to the degradation of the protein thereby decreasing the therapeutic value. Therefore, several essential approaches have been tried to enhance the stability of the protein and peptide drugs and increase absorption [208, 209]. Site-specific delivery systems, chemical modification of peptides (e.g., lipophilic derivatives, synthesis of peptidomimetics, etc.), bioadhesive 
a

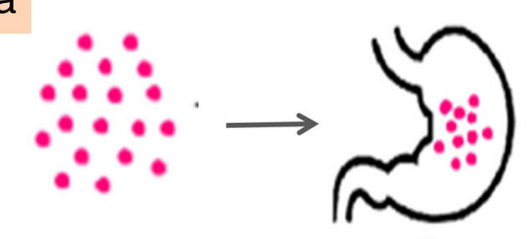

Oral peptide drug
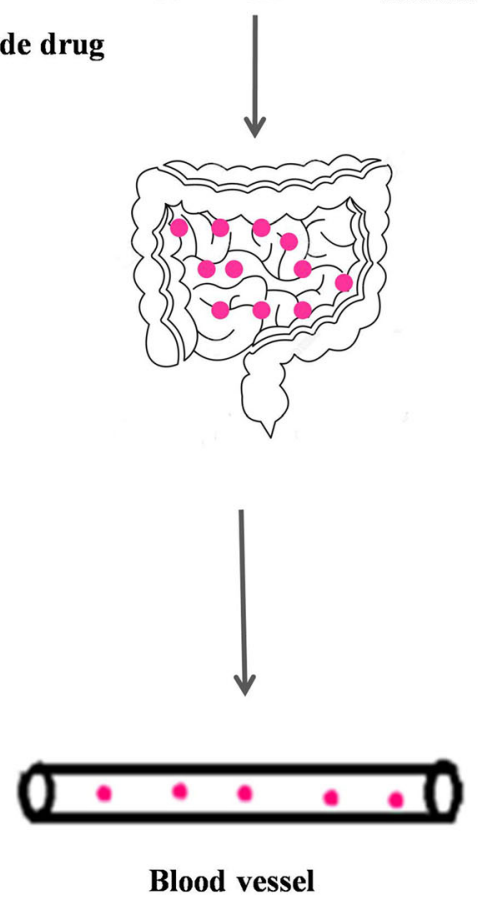

Blood vessel b

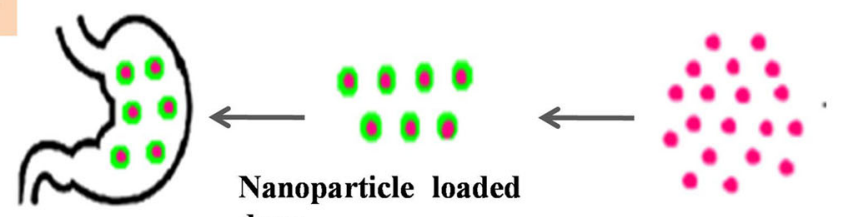

drug

Oral peptide drug
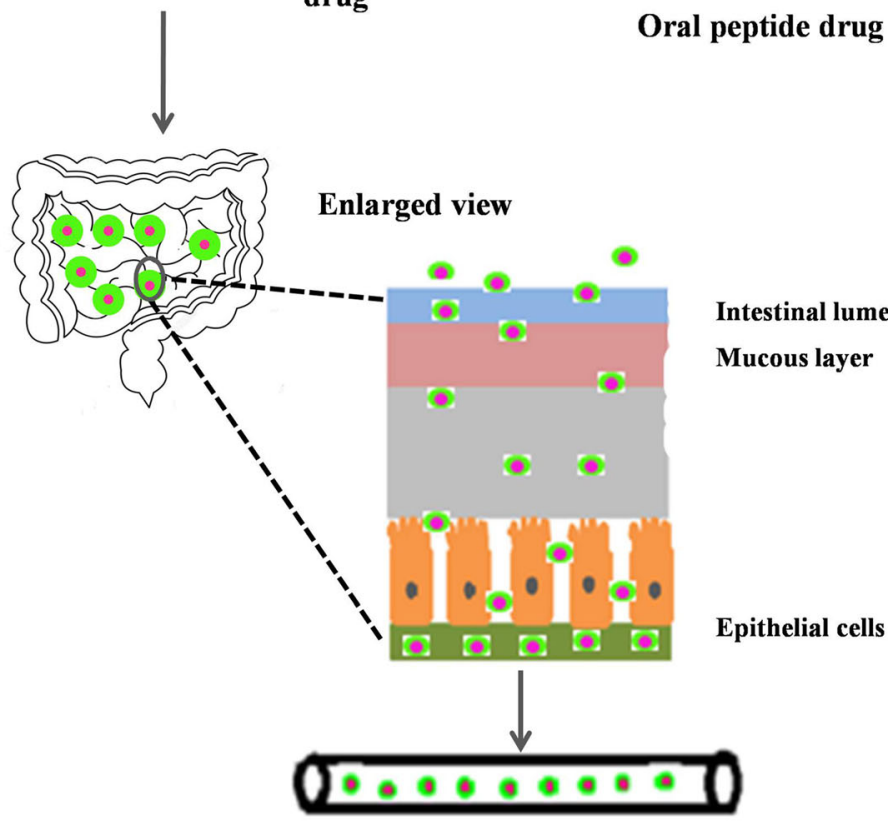

Blood vessel

Fig. 10 Administration of $\mathrm{pH}$ sensitive peptide drug via oral delivery. a The peptide drug administered orally degraded particularly in stomach due to proteolytic enzymes which result in poor availability of drugs. b The nanoparticles shields drugs and prevent from enzymatic degradation. Hence attains the efficient distribution of drugs

systems and concomitant administration of penetration enhancers or protease inhibitors have been investigated to improve the oral delivery of peptides [210]. After oral administration, the nanocarriers will encounter the physicochemical environment of the GIT. The human intestinal epithelium is composed of villi that increase the total absorptive surface area in the GIT to $300-400 \mathrm{~m}^{2}$ and acts as a physical barrier to drug absorption [138]. It is composed of absorptive enterocytes and for a large part sprinkled by mucus-producing goblet cells, endocrine, and Paneth cells. Immunocompetent cells (B and $\mathrm{T}$ lymphocytes, dendritic cells) are located in the lamina propria beneath the epithelium except for intraepithelial lymphocytes and dendritic cells that are inserted between the enterocytes. Biological fluids influence the strength of particles even before they enter and have contact with the intestinal cells.

\section{Nanotechnology in oral-drug delivery}

Nanotechnology comes with its own set of advantages in the drug delivery field, particularly in oral drug delivery.
It allows the (i) delivery of poorly water-soluble drugs, (ii) targeting of drugs to a specific part of the gastrointestinal tract, (iii) transcytosis of drugs across the tight intestinal barrier and (iv) intracellular and transcellular delivery of large macromolecules [211]. Nanoparticle encapsulation is one such method to overcome the GI barrier, protect the drug from enzymatic degradation and release them in a controlled or systemic manner [212]. Use of a biodegradable polymeric nanoparticle is another promising approach to the pre-oral delivery of protein and peptide drugs with improved drug efficacy (Fig. 10) [213-215]. Polymeric nanocarriers can protect the drugs thereby increasing the absorption rate, and the nanocarrier composition will strongly influence its stability in the GIT. If nanoparticles are prepared with insoluble polymers, they will neither be degraded nor rapidly release the drug. In contrast, water-soluble polymers which form polyelectrolyte nanoparticles will be influenced by the $\mathrm{pH}$ or ionic strength and are more likely to be destabilized. Even if their kinetic stability is better than surfactant micelles, polymeric micelles 
concentration should remain above the critical micelle concentration upon dilution in the GIT to avoid release in the GIT and should be exposed to an ionic strength below their flocculation point [160].

Transport carriers and pro-drug approaches are also used for oral drug delivery and one prime example is transferrin as a carrier to deliver insulin. Polymeric micelles have been reported to cross the intestinal barrier after oral administration and therefore it is effectively used for oral drug delivery [216]. Zhang et al combined starch nanoparticles as the backbone and poly $(\mathrm{L}$-glycolic acid) as a graft to develop a pH-responsive starch nanoparticles-g-PGA (SNP-g-PGA) that acted as a carrier to orally deliver insulin [217]. Considerable advancements are still required for the development of innovative materials and technologies to maximize drug absorption and stability in oral-drug delivery. Glucagonlike peptide-1 (GLP-1) is a small peptide hormone produced from intestinal L-cells and effective in lowering hyperglycemic conditions. Because of very short plasma half-life $(<5 \mathrm{~min})$ and rapid metabolic clearance, the anti-diabetic effect of GLP-1 could be better utilized with oral-gene delivery methods. In this context, antibody-mediated (human IgG1 (hIgG1)-Fc-Arg/pDNA complexes were prepared as an oral-gene delivery system for the prevention of type 2 diabetes mellitus (T2DM) [218].

Oral delivery of methylthioadenosine (MTA) to the brain by solid lipid nanoparticles was reported for the effective management of multiple sclerosis-like conditions in mice. As compared to plain MTA, MTA loaded SLN not only offers high drug entrapment but also increased the half-life of MTA from $28 \mathrm{~min}$ to $1.25 \mathrm{~h}$ and improve the locomotors activity from 49 to $79 \%$, respectively [219]. However, recent advancements in oral-drug delivery include the development of bioadhesive food protein nanoparticles using zein (Z) and whey protein (WP). The hydrophobic corn protein zein is used as a core and whey protein acts as a shell to deliver the antiretroviral drug Lopinavir (LPN) and fenretinide, an investigational anticancer agent. Similar to MTA loaded SLN, ZWP nanoparticles have also increased the half-life and bioavailability of both drugs when administrated orally [220]. Oral-administration routes continued to improve the therapeutic effect of peptides. However, challenges associated with antihypertensive peptides are rapid degradation and poor bioavailability. Though injections are considered an alternate routine of peptide drug administration, it results in poor patient compliance because of repeated injections. Hence, a novel oral peptide delivery system like Tyr-Gly-Leu-Phe (YF4)-loaded lipid nanoparticles (YF4-LNPs) was developed to utilize the advantage of both polymer nanoparticles and liposome. The in vitro release profile showed burst release of $80 \%$ free
YF4 within $6 \mathrm{~h}$ while YF4-LNP showed less than $40 \%$ release in $24 \mathrm{~h}$. The in vivo antihypertensive activity in the animal model showed the decrease of SBP (Systolic Blood Pressure) by $15.6 \mathrm{mmHg}$ at $4 \mathrm{~h}$ post-administration while in YF4-LNPs, blood pressure decreased by $43.5 \mathrm{mmHg}$ in about $2 \mathrm{~h}$ post-administration [221].

\section{Inhalation route}

Pulmonary delivery has several irreplaceable advantages over other delivery routes such as oral or injection. It avoids first-pass hepatic metabolism thus reducing dose requirement and side effects. Pulmonary delivery also allows local delivery of therapeutics targeting respiratory diseases such as asthma, chronic obstructive pulmonary disease (COPD), and cystic fibrosis. The pulmonary route offers other advantages such as a high surface area with rapid absorption due to high vascularization and circumvention of the first-pass effect [222]. The pulmonary route has been used for local delivery of drugs like antibiotics (cyclosporine, tobramycin, amikacin, fluoroquinolones) [223] proteins and peptides (insulin, amylin, calcitonin) [224], chemotherapeutics (doxorubicin, fluorouracil, cisplatin) $[225,226]$, interferon (interferon- $\alpha$, interferon- $\gamma$ etc) [223], and vaccines (measles, influenza, tuberculosis, hepatitis) $[227,228]$.

The lung consists of two functional parts, the airways (trachea, bronchi, and bronchioles) and the alveoli (gas exchange areas). The conducting zone consists of the first 16 generations of airways comprised of the trachea (generation 0), which separates into the two mainstem bronchi and subdivides progressively into smaller bronchi and bronchioles. The respiratory zone consists of all structures that participate in gas exchange and begins with the respiratory bronchioles [229]. The particles that are less than $5-6 \mu \mathrm{m}$ are deposited into the tracheabronchial region. Ultrafine particles $(1-2 \mu \mathrm{m})$ settle in the bronchioles and particles at the nanoscale $(<1 \mu \mathrm{m})$ are delivered into the lower respiratory system. Ultrasmall-sized nanoparticles such as dendrimers $(<20 \mathrm{~nm})$ showed efficient delivery to the alveoli but they often presented low retention in the lungs due to the rapid penetration into the bloodstream [230-232]. The most important mechanisms of particle deposition in the respiratory tract are inertial impaction, gravitational sedimentation, and diffusion (Brownian motion).

\section{Nanoparticle formulations for drug delivery to the lungs}

A challenge for nanoparticulate drug delivery to the lungs is to understand the fate of the particles and their interactions with biological systems. To successfully deliver an inhalable drug it should overcome pulmonary clearance (mucociliary escalator, alveoli) and detoxification activity of enzymes like cytochrome P450. Rapid particle clearance reduces sustained delivery of the drug 


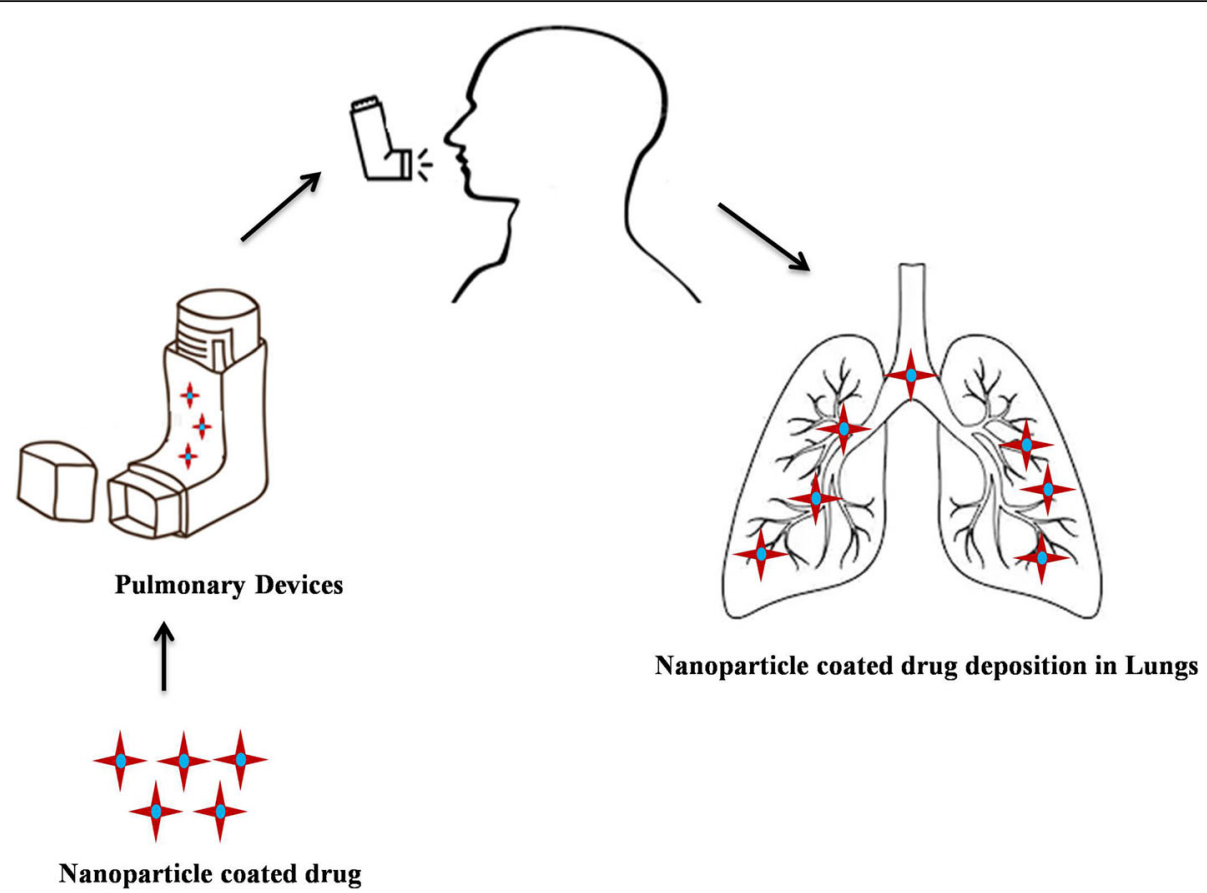

Fig. 11 Pulmonary drug delivery via inhalation. The concept of nanoparticle incorporated drugs for pulmonary delivery is, when it is inhaled it will pass through oropharynx and deposited in alveoli of lungs with the help of suitable inhalation devices. The pulmonary device containing nanoparticle coated drugs, when inhaled will pass through oropharynx and deposited in the alveoli of lungs. Subsequently, the nanoparticle coated drug aids in sustained release of drugs from the lungs and thus improved distribution in systemic circulation. It offers high surface area with rapid absorption vascularization and circumvention of the first pass effect

and particle translocation might bring nanoparticles to undesired areas of the body. To overcome these obstacles and increase efficiency, a particulate based drug delivery system is introduced. It uses carriers (liposomes, solid lipid nanoparticles, polymers, etc.) to encapsulate the drug thereby increasing half-life of the drugs [233]. Nanoparticles could provide the advantage of sustained release in the lung tissue, followed by the systemic circulation leading to a reduction in dosage frequency and improved patient compliance (Fig. 11). Nanoparticle deposition in the respiratory tract is determined predominantly by diffusional alteration due to the thermal motion of air molecules interacting with particles in the inhaled and exhaled air streams [234, 235]. Three types of pulmonary delivery devices are commercially available: 1 ) pressurized metered-dose inhalers (pMDI), 2) nebulizers and 3) dry powder inhalers (DPI). DPI and MDI make use of impaction were aerosol particles travel at high velocity settling in the oropharynx region due to centrifugal force. Sedimentation is the most important technique for the nanoparticulate system since the particle settles for a long time at the site and, as a result, increases the efficiency of the drug [222]. The aerodynamic diameter of nanoparticles is the primary determinant for in vivo distribution of the inhaled nanoparticles [236]. Depending on the particle size, shape and ventilation parameters deposition occur in all regions of the lungs (the airways and the alveoli). With decreasing particle diameter below about $500 \mathrm{~nm}$, the deposition increases in all regions of the lung because of the increasing diffusional mobility [237].

The pharmacokinetics of the nanoparticles can be altered after a structural modification. The dendrimers without any surface modification get absorbed into the bloodstream with limited lung retention, but PEGmodified dendrimers with larger sizes $(>78 \mathrm{kDa})$ will accumulate in the lungs [238]. To increase the shelf life of the drugs, they can be coated with stealth material (e.g. hyaluronic acid) which forms a hydration layer that prevents immune recognition [239]. Rifampicin is a known first-line drug for tuberculosis that exhibits selfaggregation in the aqueous phase which affects the preparation of liquid pediatric tuberculosis formulation. The self-aggregation of drug molecules is resolved by encapsulating within the commercial polymeric micelles Kolliphor ${ }^{\ominus}$ HS 15. The nanoscale Kolliphor ${ }^{\ominus}$ HS 15 micelles have improved the aqueous solubility and microbicidal activity to 14.3 fold and 2.5 fold, respectively [240]. Antiinflammatory drug, budesonide encapsulated in solid lipid nanoparticle suspension (SLNPs) was used to test the efficacy of endotracheal aerosolization (ETA) device for pulmonary delivery. In ETA, nanoparticle 


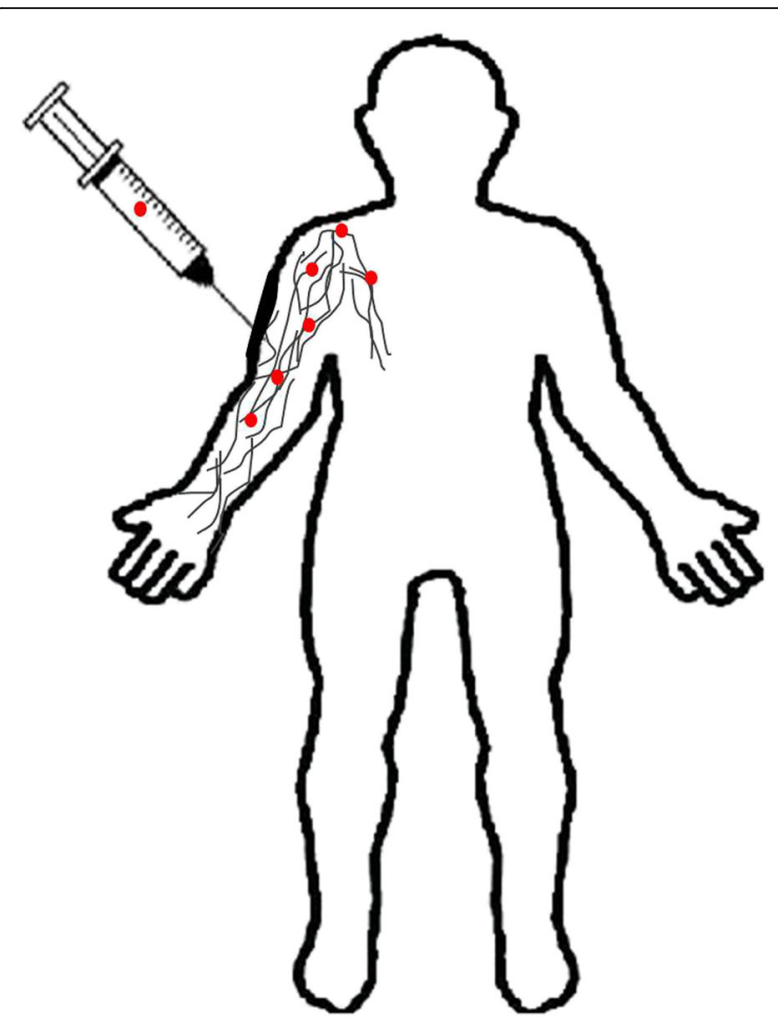

Fig. 12 Systemic delivery of nanoparticles by intravenous injection. Intravenous drug administration via blood stream is equally popular route of drug administration and offers the systemic action as well as complete bioavailability. The uncoated or raw nanoparticles have often suffers with the effect of opsonization or macrophage uptake, especially nanoparticles with $<\sim 5 \mathrm{~nm}$ rapidly undergo renal clearance upon intravenous administration. Surface tailoring is the effective way of prevent clearance and improve the cellular uptake for maximum drug accumulation in tumor sites. Nanoemulsions and micellar nanocomplex are significantly used in recent times to enhance the anti-tumor effect of the drug with infinitesimal off-target toxicity

suspensions are directly aerosolized within the trachea and readily deposited into the pulmonary region. Furthermore, it is a non-invasive and promising method with high efficiency. The budesonide loaded SLNPs formulation has shown $80 \%$ pulmonary deposition in Sprague-Dawley rats and a high in vitro emission rate [241]. Similarly, pulmonary delivery of nanocomposite microparticles (NCMPs) i.e. PGA-co-PDL nanoparticles with microRNA (miR-146a) by dry powder inhalation was useful for the treatment and management of chronic obstructive pulmonary disease (COPD) [242]. The activity of miR-146a was preserved after the spray-drying process and miR-146a loaded NCMPs were used to silence the target genes IRAK1 and TRAF6. MiR-146a-5p demonstrated its protective effects against tumorigenesis and development of diverse neoplasms, including non-small cell lung cancer (NSCLC) by down-regulating the IRAK1 (IL-1 receptor-associated kinase 1) and TRAF6 (TNF receptor-associated factor 6) expression [243].

\section{Intravenous delivery}

Nanoparticles can be administered through different routes including intravenous and intraperitoneal injection, oral administration, and pulmonary inhalation. The IV route provides almost instantaneous response and allows wide-ranging control of the rate of drug contribution into the body. It is also suitable for drugs which cannot be absorbed by the gastrointestinal tract or which cannot be injected into muscles or other tissues, equally important it overcomes the problem of first-pass metabolism [244]. Expensive drugs such as peptides and proteins are delivered efficiently by intravenous route. Intravenous administration overcomes the degradation by proteolytic enzymes (Fig. 12). The main advantage of intravenous drug delivery is the rapid onset of action and complete bioavailability of drugs even with low doses. There are many risks associated with IV route because of the direct exposure of the drug in the systemic circulation. It is painful for the patient, expensive and requires the assistance of experienced healthcare personnel. The first intravenously administered nanoparticulate product, Abraxane ${ }^{\circ}$ (a reformulation of paclitaxel), was approved by the FDA in 2006 [245, 246].

The major difficulties in current cancer therapy are mostly the drug side effects due to drug accumulation, cancer recurrence, and delay in disease stabilization. These challenges can be overcome by nanomedicines. Clinical trials in humans demonstrated that controlled release nanocarriers can be intravenously infused and guided towards local tumor site that in turn augment the efficacy of solid tumors treatment. It reduces the toxic side effects of the drugs and produces prolonged remission. Drug polymer conjugates and nanoemulsions are mainly explored for targeting prostate cancer [247]. Paclitaxel is a first-line chemotherapy drug which is commercially available as paclitaxel-cremophor (1:1) combination. Paclitaxel, when administered with cholesterol-rich nanoemulsion (LDE), had displayed low toxicity and increased anticancer activity in a mouse model. Further, LDE tends to concentrate on solid tumors and binds to cancer cells overexpressing LDL receptors. Recently, the pharmacokinetic and tumor uptake efficiency of paclitaxel-LDE and paclitaxelcremophor was compared in human gynecological cancers. The mean half-life of paclitaxel-cremophor were $6.62 \pm 2.05 \mathrm{~h}$ whereas paclitaxel-LDE has shown T1/2 of $14.51 \pm 3.23 \mathrm{~h}$, and it also showed higher targeting in tumor tissues (3.5times) than normal tissues [248].

At present, various studies have been conducted on the delivery of nanoparticle-associated drugs by the intravenous route. The nanoparticle is not able to 
efficiently deliver drugs due to RES uptake. To overcome this problem, surface modification of nanoparticles can be carried out. Xiang et al developed SLNP-containing dexamethasone acetate (DXM). DXM alone and DXMSLNP are intravenously administered to mice. For DXM-SLNP and DXM, the biodistribution showed a significantly different pattern. The area under the drug concentration-time curve of DXM-SLNP in the lung was 17.8-fold larger in comparison to that of DXM solution alone [249]. For the intravenous application of emulsions, the size of oil droplet should be below the size of the smallest blood vessel in the lungs which is $5 \mu \mathrm{m}$. The mean droplet size of these particles is in the range of $200-400 \mathrm{~nm}$ and is consequently called nanoemulsion [250]. Though iron has been used to treat anemia for more than 300 years, oral iron therapy invariably results in gastrointestinal toxicity and takes a long time to combat the disease.

Intravenous delivery overcomes this limitation to some extent with fewer side effects and rapid release of iron. Third generation IV-iron therapies have especially improved the efficacy significantly without any toxicity issues encountered during the old-generation iron therapy. The accelerated dose of Cosmofer (iron dextran) administration has proven to be effective, very safe, time-saving and it enhances the reduction in nursing time without any late adverse reaction for the chronic kidney disease (CKD) patients [251]. As of now, the best-developed IV formulation comprises iron-oxyhydroxide core encompassed within carbohydrate shells of different sizes and polysaccharide branches. However, the toxicity profiles should be evaluated because longterm clinical use is widespread [252]. A new micellar nanocomplex consisting of IONP conjugated HA was fabricated to deliver the drug homocamptothecin (HCPT) via intravenous administration. The combined magnetic and CD44 binding ability from IONP and HA, respectively have ensured increased uptake and theranostic potency of HA-IONP/HCPT (HIH) in human squamous cell carcinoma cell line (SCC-7 cells) through superior EPR permeability retention targeting. The administration of $3 \mathrm{mg} / \mathrm{kg}$ of $\mathrm{HIH}$ in the presence of a magnetic field showed complete disappearance of the tumor after 14 days in mice model. The results demonstrated the translational potential of HIH nanocomplex for cancer theranostics owing to its excellent tumor targeting ablation with no systemic toxicity [253].

\section{Nanotoxicity}

Nanoparticle drug delivery offers enormous benefits due to it highly stable nature and its ability to encapsulate both hydrophilic and hydrophobic substances. Importantly, nanoparticles are consistent with various routes of administration [156]. Addressing nanoparticle drug delivery nanotoxicity is of great significance. As of now (July 2019) PubMed has enlisted 43,570 and 21,835 articles for the search terms "nanoparticle drug delivery" and "nanoparticle toxicity", respectively. The unique properties of nanoparticles such as the small surface to volume ratio are alluring and possibly valuable from an engineering or biomedical point of view. Likewise, the properties that may give rise to unexpected toxicities are equally interesting [254]. The toxicity level of anionic nanoparticles are considerably less toxic; whereas the cationic nanoparticles like gold and polystyrene nanoparticles have been reported to cause hemolysis and clotting [110].

Nanomaterials can enter the body through several routes including the skin, respiratory tract, parenteral administration, etc. In the blood, it will come in contact with plasma proteins that will probably lead to the formation of protein corona which may modify the pharmacological properties of the nanoparticles. The interaction between the nanoparticle and the body should be properly assessed since toxicity is of great concern [255]. In vivo and in vitro studies of nanoparticles have shown that the minor toxicities observed are due to increasing ROS levels and disruption of the host homeostasis [256]. The ROS could further damage the genome and create oxidative stress conditions that in turn induce micronuclei formation. Irrespective of their size, amorphous $\mathrm{TiO}_{2}(30 \mathrm{~nm})$ and silver nanoparticles $(15 \mathrm{~nm})$ induce the highest generation of reactive oxygen species. The possible engulfment of silver nanoparticles and quantum dots by macrophages certainly will enhance the expression of inflammatory mediators TNF- $\alpha$, MIP-2 and IL-1 $\beta$, irrespective of their size [257]. Nanoparticles tend to accumulate in the liver, therefore the detailed mechanism of how these particles are eliminated from the body should be investigated [156]. A single and multi-walled carbon nanotube induces platelet aggregation whereas their building blocks C-60 fullerenes do not. The principle behind nanomaterial functioning will be addressed in detail for successful and safe drug delivery. The use of nanoparticles is increasing. Similarly, toxicity issues must also be considered.

\section{Conclusions}

This analysis provides an overview of the different nanocarriers/NPs and various routes of drug administration for improved drug delivery along with detailing the challenges associated with the nanocarrier systems. With the help of cutting-edge technology, a variety of natural and synthetic polymers have been successfully engineered to deliver drugs with improved efficiency. Though nanoparticles offer higher drug loading, better bioavailability, etc., nanoparticle-mediated toxicity is yet to be resolved to satisfaction. Hence, extensive research and 
development is currently focused on initiating controlled drug delivery with less toxicity. Polymers like chitosan are commonly used for drug delivery owing to their biodegradable, biocompatible and mucoadhesive properties. For the past decennium, the concept of biomimetic has been introduced in material design to create more biologically attractive nanocarriers. This could either be achieved by introducing suitable ligands to the CNT surface or by fabricating chitosan nanoparticle with the desired chemical molecule or moiety promoting selfassembly for increased cellular uptake. The successful delivery of a drug to the target region requires not only an ideal nanocarrier but also an effective route of drug administration that enables crossing the blood-brain barrier. However, each route of administration has its advantages and disadvantages when it comes to targeted drug delivery. To overcome the limitations of different administration routes, superior understanding of intercellular, transcellular and other carrier-mediated transporting pathways are essential to develop the next-generation of futuristic nanocarriers. The creation of such an advanced nanotherapeutic system will mark the beginning of a new era in nanotechnology-based drug delivery.

\section{Abbreviations}

5-ASA: 5-Aminosalicylic acid; 5-FU: 5-Fluorouracil; AA: Acrylic acid; AJs: Adherens Junctions; ALL: Acute Lymphoblastic Leukemia; AUC: Area under the Curve; AZ-HECTS: Azidehydroxyethyl chitosan; BBB: Blood-brain barrier; BML: Bubble-generating magnetic liposomal drug delivery system BSA: Serum Albumin; CKD: Chronic Kidney Disease; CLSM: Confocal Laser Scanning Microscopy; CNS: Central Nervous System; CNT: Carbon Nanotubes; COPD: Chronic Obstructive Pulmonary Disease; CQDs: Carbon Quantum Dots; CT: Computed Tomography; DDS: Drug Delivery System; DMSA: Dimercaptosuccinic acid; DOX: Doxorubucin; DPI: Dry Power Inhalers; DXM: Dexamethasone acetate; EA: Ellagic acid; EMA: European Medicine Agency; EPR: Enhanced Permeability and Retention; ETA: Endotracheal aerosolization; FA: Folic acid; FAE: Follicle associated epithelium; FDA: US Food and Drug Administration; GALT: Gut Associated Lymphoid Tissue; GIT: Gastrointestinal tract; GLP-1: Glucagon like peptide; GMO-MNPs: Glycerol mono-oleate-coated magnetic nanoparticles; HA: Hyaluronic acid; HC: Hydroxyethylacryl Chitosan; HCPT: 10-hydroxy camptothecin/ homocamptothecin; HER2: Human epidermal growth factor receptor 2; HIH: HA-IONP/HCPT; IG: Indocyanine green; IL: Interleukin; IONPs: Iron Oxide Nanoparticle; IR: Insulin Receptor; LDE: Cholesterol-rich nanoemulsion; LDL: Low Density Lipoprotein; LMWC: Low Molecular Weight Chitosan; LPN: Lopinavir; MDR: Multidrug Resistance; MIP: Macrophage-Inhibitory Protein; MMP: Matrix Metalloproteinases; MNPs: Magnetic nanoparticles; MPS: Mononuclear Phagocyte System; MRI: Magnetic Resonance Imaging; MTA: Methylthioadensine; MW: Molecular Weight; MWCNT: Multi-wall Carbon Nanotubes; nAChR: nicotinic Acetylcholine Receptor; NC: Nanocarriers; NIR: Near-infrared; NPs: Nanoparticles; OSC: Organic Solar Cells; PAF: Plateletactivating factor; PAMAM: Polyamidoamine; PCL: Polycaprolactones: PDT: Photodynamic therapy; PE: Phosphoethanolamine; PEG: Polyethylene glycol; PEGMEA: PEG methyl-ether acrylate; PEl: Polyethyleneimine; PET: PositronEmission Tomography; P-gp: P-glycoprotein; PLA: Polylactic acid; PLA2: Phospholipase A2; PLGA: Poly(lactic-co-glycolic acid); PLL: Poly(L-lysine); pMDI: pressurized Metered Dose Inhalers; PMMA: Poly(methyl methacrylate); pNIPA: poly(N-isopropyl acrylamide); PPI: Poly(propylene imine); PTT: Photothermal therapy; PTX: Paclitaxel; QDs: Quantum Dots, rdAds: replication-defective adenoviruses; RES: Reticuloendothelial system ROS: Reactive Oxygen Species; SA: Sodium Alginate; SBP: Systolic blood pressure; SLNP: Solid Lipid Nanoparticles; SMANCS: Styrene maleic anhydrideneocarzinostatin; SNP-g-PGA: Starch nanoparticles-poly(L-Glycolic acid); SPM: Superparamagnetic; SPR: Surface Plasmon Resonance; SWCNT: Single
Wall Carbon Nanotubes; TDD: Transdermal Drug Delivery; TfR: Transferrin Receptor; TJs: Tight Junctions; TMC: Trimethyl Chitosan; TNF-a: Tumor Necrosis Factor-a; W: Whey protein; Z: Zein; ZWP: Zein whey protein

\section{Acknowledgments}

The co-author Dr. Sadhasivam Subramaniam acknowledges DBT, Government of India for the financial support provided by the Ramalingaswami Re-entry Fellowship (Order No. BT/RLF/Re-entry/55/2013). Other authors also wish to thank their respective institutions for their continued support. The authors declare no conflict of interest. The Qatar National Library (QNL), Doha, Qatar, funded the publication of this article.

\section{Authors' contributions}

DC, SGR, SK and FHL performed extensive research and collected information and generated short write-ups. SS, MME and MWQ contributed to conceptual work, framework, draft write-up, editing and critical evaluation. All authors read and approved the final manuscript.

\section{Funding}

This research received no specific grant from any funding agency in the public, commercial, or not-for-profit sectors.

\section{Availability of data and materials}

This is a review article. All data generated or analyzed during this study are included in this published article.

Ethics approval and consent to participate

Not applicable.

\section{Consent for publication}

Not applicable.

\section{Competing interests}

The authors declare that they have no competing interests.

\section{Author details}

${ }^{1}$ Department of Microbial Biotechnology, Bioprocess and Biomaterials Laboratory, Bharathiar University, Coimbatore, India. ${ }^{2}$ Department of Extension and Career Guidance, Bharathiar University, Coimbatore, India. ${ }^{3}$ Department of Food Science and Nutrition, College of Agricultural and Marine Sciences, Sultan Qaboos University, Muscat, Oman. ${ }^{4}$ Institute of Biomedical Engineering and Nanomedicine, NationalHealth Research Institutes, Miaoli, Taiwan. ${ }^{5}$ Research and Policy Department, World Innovation Summit for Health (WISH), Qatar Foundation, P.O. Box 5825, Doha, Qatar.

Received: 1 July 2019 Accepted: 20 September 2019

Published online: 21 November 2019

References

1. Tarafdar JC, Sharma S, Raliya R. Nanotechnology: Interdisciplinary science of applications. Afr J Biotechnol. 2013;12(3):219-26.

2. Muller RH, Keck CM. Challenges and solutions for the delivery of biotech drugs-a review of drug nanocrystal technology and lipid nanoparticles. J Biotechnol. 2004;113(1):151-70.

3. Yhee JY, Son S, Kim N, Choi K, Kwon IC. Theranostic applications of organic nanoparticles for cancer treatment. MRS Bull. 2014;39(3):239-49.

4. Kim T, Hyeon T. Applications of inorganic nanoparticles as therapeutic agents. Nanotechnology. 2013;25(1):012001.

5. Kumar S, Dilbaghi N, Saharan R, Bhanjana G. Nanotechnology as emerging tool for enhancing solubility of poorly water-soluble drugs. Bionanoscience. 2012;2(4):227-50.

6. Torchilin $\mathrm{V}$. Tumor delivery of macromolecular drugs based on the EPR effect. Adv Drug Deliv Rev. 2011;63(3):131-5.

7. Bamrungsap S, Zhao Z, Chen T, Wang L, Li C, Fu T, Tan W, et al. Nanotechnology in therapeutics: a focus on nanoparticles as a drug delivery system. Nanomedicine. 2012:7(8):1253-71.

8. Soppimath KS, Aminabhavi TM, Kulkarni AR, Rudzinski WE. Biodegradable polymeric nanoparticles as drug delivery devices. J Control Release. 2001; 70(1-2):1-20.

9. Liu Z, Jiao Y, Wang Y, Zhou C, Zhang Z. Polysaccharides-based nanoparticles as drug delivery systems. Adv Drug Deliv Rev. 2008;60(15):1650-62. 
10. Lü JM, Wang X, Marin-Muller C, Wang H, Lin PH, Yao Q, Chen C, et al. Current advances in research and clinical applications of PLGA-based nanotechnology. Expert Rev Mol Diagn. 2009;9(4):325-41.

11. Andonova V. Synthetic Polymer-Based Nanoparticles: Intelligent Drug Delivery Systems. In: Boreddy SR, editor. Acrylic Polymers in Healthcare. Reddy: Intech Open; 2017. p. 101-25.

12. M Ways T, Lau W, Khutoryanskiy V. Chitosan and its derivatives for application in mucoadhesive drug delivery systems. Polymers. 2018;10(3): 267.

13. Sajomsang W, Ruktanonchai UR, Gonil P, Nuchuchua O. Mucoadhesive property and biocompatibility of methylated $\mathrm{N}$-aryl chitosan derivatives. Carbohydr Polym. 2009;78(4):945-52.

14. Thanou M, Florea BI, Langemeyer MW, Verhoef JC, Junginger HE. Ntrimethylated chitosan chloride (TMC) improves the intestinal permeation of the peptide drug buserelin in vitro (Caco-2 cells) and in vivo (rats). Pharm Res. 2000;17(1):27-31.

15. Gatti TH, Eloy JO, Ferreira LM, Silva IC, Pavan FR, Gremião MP, Chorilli M. Insulin-loaded polymeric mucoadhesive nanoparticles: development, characterization and cytotoxicity evaluation. Braz J Pharm Sci. 2018:54:1-10.

16. Calvo P, Gouritin B, Villarroya H, Eclancher F, Giannavola C, Klein C, Andreux $J$, Couvreur P, et al. Quantification and localization of PEGylated polycyanoacrylate nanoparticles in brain and spinal cord during experimental allergic encephalomyelitis in the rat. EJN. 2002;15(8):1317-26.

17. Faraji AH, Wipf P. Nanoparticles in cellular drug delivery. Bioorg Med Chem. 2009;17(8):2950-62.

18. Athanasiou KA, Niederauer GG, Agrawal CM. Sterilization, toxicity, biocompatibility and clinical applications of polylactic acid/polyglycolic acid copolymers. Biomaterials. 1996;17(2):93-102.

19. Tosi G, Bortot B, Ruozi B, Dolcetta D, Vandelli MA, Forni F, Severini GM, et al. Potential use of polymeric nanoparticles for drug delivery across the bloodbrain barrier. Curr Med Chem. 2013;20(17):2212-25.

20. Badri W, Eddabra R, Fessi H, Elaissari A. Biodegradable polymer based nanoparticles: dermal and transdermal drug delivery. J Colloid Sci. 2014;3(2): $141-9$.

21. Kumari A, Yadav SK, Yadav SC. Biodegradable polymeric nanoparticles based drug delivery systems. Colloids Surf B Biointerfaces. 2010;75(1):1-8.

22. Hedley ML, Curley J, Urban R. Microspheres containing plasmid-encoded antigens elicit cytotoxic T-cell responses. Nat Med. 1998;4(3):365-8.

23. Panyam J, Labhasetwar $V$. Biodegradable nanoparticles for drug and gene delivery to cells and tissue. Adv Drug Deliv Rev. 2003:55(3):329-47.

24. Fadel M, Kassab K, Fadeel DA. Zinc phthalocyanine-loaded PLGA biodegradable nanoparticles for photodynamic therapy in tumor-bearing mice. Lasers Med Sci. 2010;25(2):283-92.

25. Mohammed M, Syeda J, Wasan K, Wasan E. An overview of chitosan nanoparticles and its application in non-parenteral drug delivery. Pharmaceutics. 2017;9(4):53.

26. Raftery R, O'Brien F, Cryan SA. Chitosan for gene delivery and orthopedic tissue engineering applications. Molecules. 2013;18(5):5611-47.

27. Janes KA, Calvo P, Alonso MJ. Polysaccharide colloidal particles as delivery systems for macromolecules. Adv Drug Deliv Rev. 2001;47(1):83-97.

28. Patel MP, Patel RR, Patel JK. Chitosan mediated targeted drug delivery system: a review. J Pharm Pharm Sci. 2010;13(4):536-57.

29. Tozaki H, Odoriba T, Okada N, Fujita T, Terabe A, Suzuki T, Okabe S, Muranishi S, Yamamoto A, et al. Chitosan capsules for colon-specific drug delivery: enhanced localization of 5-aminosalicylic acid in the large intestine accelerates healing of TNBS-induced colitis in rats. J Control Release. 2002; 82(1):51-61.

30. Rodrigues LB, Leite HF, Yoshida MI, Saliba JB, Junior AS, Faraco AA, et al. In vitro release and characterization of chitosan films as dexamethasone carrier. Int J Pharm. 2009;368(1-2):1-6.

31. Yuan ZX, Sun X, Gong T, Ding H, Fu Y, Zhang ZR, et al. Randomly 50\% Nacetylated low molecular weight chitosan as a novel renal targeting carrier. J Drug Target. 2007;15(4):269-78.

32. Pandey H, Rani R, Agarwal V. Liposome and their applications in cancer therapy. Braz Arch Biol Technol. 2016;59:e16150477.

33. Mishra V, Bansal K, Verma A, Yadav N, Thakur S, Sudhakar K, Rosenholm J. Solid lipid nanoparticles: emerging colloidal nano drug delivery systems. Pharmaceutics. 2018;10(4):191.

34. Uchechi O, Ogbonna JD, Attama AA. Nanoparticles for dermal and transdermal drug delivery. In: Application of Nanotechnology in Drug Delivery. Ali Demir Sezer: InTech C; 2014. p. 193-235.
35. Xue M, Yang MX, Zhang W, Li XM, Gao DH, Ou ZM, Li ZP, Liu SH, Li XJ, Yang SY, et al. Characterization, pharmacokinetics, and hypoglycemic effect of berberine loaded solid lipid nanoparticles. Int J Nanomedicine. 2013:8:4677.

36. Chen R, Wang S, Zhang J, Chen M, Wang Y. Aloe-emodin loaded solid lipid nanoparticles: formulation design and in vitro anti-cancer study. Drug deliv. 2015;22(5):666-74

37. Baek JS, Cho CW. Surface modification of solid lipid nanoparticles for oral delivery of curcumin: Improvement of bioavailability through enhanced cellular uptake, and lymphatic uptake. Eur J Pharm Biopharm. 2017;117:132-40.

38. Svenson S. The dendrimer paradox-high medical expectations but poor clinical translation. Chem Soc Rev. 2015;44(12):4131-44.

39. Labieniec-Watala M, Watala C. PAMAM dendrimers: destined for success or doomed to fail? Plain and modified PAMAM dendrimers in the context of biomedical applications. J Pharm Sci. 2015;104(1):2-14

40. Jain K, Kesharwani P, Gupta U, Jain NK. Dendrimer toxicity: Let's meet the challenge. Int J Pharm. 2010:394(1-2):122-42.

41. Nassimi M, Schleh C, Lauenstein HD, Hussein R, Hoymann HG, Koch W, Pohlmann GA, Krug N, Sewald K, Rittinghausen S, Braun A, et al. A toxicological evaluation of inhaled solid lipid nanoparticles used as a potential drug delivery system for the lung. Eur J Pharm Biopharm. 2010; 75(2):107-16.

42. Elhissi AM, Islam MA, Arafat B, Taylor M, Ahmed W. Development and characterisation of freeze-dried liposomes containing two anti-asthma drugs. Micro Nano Lett. 2010;5(3):184-8.

43. Hoesel LM, Flierl MA, Niederbichler AD, Rittirsch D, McClintock SD, Reuben JS, Pianko MJ, Stone W, Yang H, Smith M, Sarma JV, et al. Ability of antioxidant liposomes to prevent acute and progressive pulmonary injury. Antioxid Redox Signal. 2008;10(5):963-72.

44. Tripathy S, Das MK. Dendrimers and their applications as novel drug delivery carriers. J Appl Pharm Sci. 2013;3(09):142-9.

45. Mignani S, Rodrigues J, Tomas H, Caminade AM, Laurent R, Shi X, Majoral JP, et al. Recent therapeutic applications of the theranostic principle with dendrimers in oncology. SCMs. 2018;61(11):1367-86.

46. Jevprasesphant R, Penny J, Jalal R, Attwood D, McKeown NB, D'emanuele A, et al. The influence of surface modification on the cytotoxicity of PAMAM dendrimers. Int J Pharm. 2003;252(1-2):263-6.

47. Bhadra D, Bhadra S, Jain S, Jain NK. A PEGylated dendritic nanoparticulate carrier of fluorouracil. Int J Pharm. 2003;257(1-2):111-24.

48. Waite $\mathrm{CL}$, Sparks SM, Uhrich KE, Roth CM. Acetylation of PAMAM dendrimers for cellular delivery of siRNA. BMC Biotechnol. 2009;9(1):38.

49. Palmerston Mendes L, Pan J, Torchilin V. Dendrimers as nanocarriers for nucleic acid and drug delivery in cancer therapy. Molecules. 2017;22(9):1401.

50. Qiao Z, Shi X. Dendrimer-based molecular imaging contrast agents. Prog Poly Sci. 2015;44:1-27.

51. Leiro V, Garcia JP, Tomas H, Pego AP. The present and the future of degradable dendrimers and derivatives in theranostics. Bioconjug Chem. 2015;26(7):1182-97.

52. Xu $R$, Wang $Y$, Wang $X$, Jeong EK, Parker $D L$, Lu ZR, et al. In vivo evaluation of a PAMAM-cystamine-(Gd-DO3A) conjugate as a biodegradable macromolecular MRI contrast agent. Exp Biol Med. 2007;232(8):1081-9.

53. Mekuria SL, Debele TA, Tsai HC. Encapsulation of gadolinium oxide nanoparticle $\left(\mathrm{Gd}_{2} \mathrm{O}_{3}\right)$ contrasting agents in PAMAM dendrimer templates for enhanced magnetic resonance imaging in vivo. ACS Appl Mater Interfaces. 2017:9(8):6782-95.

54. Jędrzak A, Grześkowiak BF, Coy E, Wojnarowicz J, Szutkowski K, Jurga S, Jesionowski T, Mrówczyński R, et al. Dendrimer based theranostic nanostructures for combined chemo-and photothermal therapy of liver cancer cells in vitro. Colloids Surf B Biointerfaces. 2019;173:698-708.

55. Lu M, Liu Y, Huang YC, Huang CJ, Tsai WB. Fabrication of photocrosslinkable glycol chitosan hydrogel as a tissue adhesive. Carbohydr Polym. 2018;181:668-74.

56. Khanmohammadi M, Sakai S, Taya M. Fabrication of single and bundled filament-like tissues using biodegradable hyaluronic acid-based hollow hydrogel fibers. Int J Biol Macromol. 2017;104:204-12.

57. Capanema NS, Mansur AA, de Jesus AC, Carvalho SM, de Oliveira LC, Mansur HS, et al. Superabsorbent crosslinkedcarboxymethyl cellulose-PEG hydrogels for potential wound dressing applications. Int J Biol Macromol. 2018;106:1218-34

58. Farhat W, Venditti R, Mignard N, Taha M, Becquart F, Ayoub A, et al. Polysaccharides and lignin based hydrogels with potential pharmaceutical 
use as a drug delivery system produced by a reactive extrusion process. Int J Biol Macromol. 2017;104:564-75.

59. El Fawal GF, Abu-Serie MM, Hassan MA, Elnouby MS. Hydroxyethyl cellulose hydrogel for wound dressing: fabrication, characterization and in vitro evaluation. Int J Biol Macromol. 2018;111:649-59.

60. Li JF, Liu WS, Zhao LD, Zhou M. High-performance nanostructured thermoelectric materials. NPG Asia Mater. 2010;2(4):152-8.

61. He M, Han B, Jiang Z, Yang Y, Peng Y, Liu W, et al. Synthesis of a chitosanbased photo-sensitive hydrogel and its biocompatibility and biodegradability. Carbohydr Polym. 2017;166:228-35.

62. Wu C, Li R, Yin Y, Wang J, Zhang L, Zhong W, et al. Redox-responsive supramolecular hydrogel based on 10-hydroxy camptothecin-peptide covalent conjugates with high loading capacity for drug delivery. Mater Sci Eng C. 2017;76:196-202.

63. Osman A, Oner ET, Eroglu MS. Novel Levan and pNIPA temperature sensitive hydrogels for 5-ASA controlled release. Carbohydr Polym. 2017;165:61-70.

64. Treenate $\mathrm{P}$, Monvisade $\mathrm{P}$. In vitro drug release profiles of $\mathrm{pH}$-sensitive hydroxyethylacryl chitosan/sodium alginate hydrogels using paracetamol as a soluble model drug. Int J Biol Macromol. 2017;99:71-8.

65. D'arrigo G, Navarro G, Di Meo C, Matricardi P, Torchilin V. Gellan gum nanohydrogel containing anti-inflammatory and anti-cancer drugs: a multidrug delivery system for a combination therapy in cancer treatment. Eur J Pharm Biopharm. 2014;87(1):208-16.

66. Dalwadi C, Patel G. Thermosensitive nanohydrogel of 5-fluorouracil for head and neck cancer: preparation, characterization and cytotoxicity assay. Int J Nanomedicine. 2018;13:31-3.

67. Cherukula K, Manickavasagam Lekshmi K, Uthaman S, Cho K, Cho CS, Park IK, et al. Multifunctional inorganic nanoparticles: recent progress in thermal therapy and imaging. Nanomaterials. 2016;6(4):76.

68. Sahu A, Lee $J$ H, Lee HG, Jeong YY, Tae G. Prussian blue/serum albumin/ indocyanine green as a multifunctional nanotheranostic agent for bimodal imaging guided laser mediated combinatorial phototherapy. J Control Release. 2016;236:90-9.

69. Ma M, Chen H, Chen Y, Wang X, Chen F, Cui X, Shi J, et al. Au capped magnetic core/mesoporous silica shell nanoparticles for combined photothermo-/chemo-therapy and multimodal imaging. Biomaterials. 2012; 33(3):989-98.

70. Lu H, Wang J, Wang T, Zhong J, Bao Y, Hao H. Recent progress on nanostructures for drug delivery applications. JON. 2016;2016:20.

71. Núñez C, Estévez SV, del Pilar Chantada M. Inorganic nanoparticles in diagnosis and treatment of breast cancer. JBIC. 2018;23(3):331-45.

72. Yang HY, Li Y, Lee DS. Multifunctional and stimuli-responsive magnetic nanoparticle-based delivery Systems for Biomedical Applications. Adv Ther. 2018;1(2):1800011

73. Ito A, Shinkai M, Honda H, Kobayashi T. Medical application of functionalized magnetic nanoparticles. J Biosci Bioeng. 2005;100(1):1-1.

74. Cardoso VF, Francesko A, Ribeiro C, Bañobre-López M, Martins P, LancerosMendez S, et al. Advances in magnetic nanoparticles for biomedical applications. Adv Healthc Mater. 2018;7(5):1700845.

75. Arruebo M, Fernández-Pacheco R, Ibarra MR, Santamaría J. Magnetic nanoparticles for drug delivery. Nano Today. 2007;2(3):22-32.

76. Dilnawaz F, Singh A, Mohanty C, Sahoo SK. Dual drug loaded superparamagnetic iron oxide nanoparticles for targeted cancer therapy. Biomaterials. 2010;31(13):3694-706.

77. Martincic M, Tobias G. Filled carbon nanotubes in biomedical imaging and drug delivery. Expert Opin Drug Deliv. 2015;12(4):563-81.

78. Jain S, Singh SR, Pillai S. Toxicity issues related to biomedical applications of carbon nanotubes. J Nanomed Nanotechol. 2012;3(140):2.

79. Khodabandehloo H, Zahednasab H, Hafez AA. Nanocarriers usage for drug delivery in cancer therapy. Iran J Cancer Prev. 2016;9(2):e3966.

80. Karimi M, Solati N, Ghasemi A, Estiar MA, Hashemkhani M, Kiani P, Mohamed E, Saeidi A, Taheri M, Avci P, Aref AR, et al. Carbon nanotubes part II: a remarkable carrier for drug and gene delivery. Expert Opin Drug Deliv. 2015;12(7):1089-105.

81. Kushwaha SK, Ghoshal S, Rai AK, Singh S. Carbon nanotubes as a novel drug delivery system for anticancer therapy: a review. Braz J Pharm Sci. 2013; 49(4):629-43.

82. Son $\mathrm{KH}$, Hong JH, Lee JW. Carbon nanotubes as cancer therapeutic carriers and mediators. Int J Nanomedicine. 2016;11:5163.

83. Siu KS, Zhang Y, Zheng X, Koropatnick J, Min WP. Non-covalently functionalized of single-walled carbon nanotubes by DSPE-PEG-PEI for
SiRNA delivery. In: SiRNA delivery methods. New York: Humana Press; 2016. p. 151-63.

84. Fabbro A, Prato M, Ballerini L. Carbon nanotubes in neuroregeneration and repair. Adv Drug Deliv Rev. 2013;65(15):2034-44.

85. Keefer EW, Botterman BR, Romero MI, Rossi AF, Gross GW. Carbon nanotube coating improves neuronal recordings. Nat Nanotech. 2008;3(7):434.

86. Béduer A, Seichepine F, Flahaut E, Loubinoux I, Vaysse L, Vieu C, et al. Elucidation of the role of carbon nanotube patterns on the development of cultured neuronal cells. Langmuir. 2012;28(50):17363-71.

87. Dey NS, Rao MB. Quantum dot: Novel carrier for drug delivery. Int J Res Pharm Biomed Sci. 2011;2:448-58.

88. Zrazhevskiy P, Sena M, Gao X. Designing multifunctional quantum dots for bioimaging, detection, and drug delivery. Chem Soc Rev. 2010;39(11):4326-54.

89. Lim SY, Shen W, Gao Z. Carbon quantum dots and their applications. Chem Soc Rev. 2015:44(1):362-81.

90. Wu Y, Ali MR, Chen K, Fang N. El-Sayed MA. Nano Today: Gold nanoparticles in biological optical imaging; 2019.

91. Nedyalkov N, Koleva ME, Nikov R, Stankova NE, lordanova E, Yankov G, Aleksandrov $L$, lordanova $R$, et al. Tuning optical properties of noble metal nanoparticle-composed glasses by laser radiation. Appl Surf Sci. 2019;463:968-75.

92. Kim Y, Kim Y, Choi J, Kang T, Choi I. Determination of nanomolar levels of reactive oxygen species in microorganisms and aquatic environments using a single nanoparticle-based optical sensor. Anal Chim Acta. 2017:967:85-92.

93. Yang G, Liu J, Wu Y, Feng L, Liu Z. Near-infrared-light responsive nanoscale drug delivery systems for cancer treatment. Coordin Chem Rev. 2016;320:100-17.

94. Oh Y, Je JY, Moorthy MS, Seo H, Cho WH. pH and NIR-light-responsive magnetic iron oxide nanoparticles for mitochondria-mediated apoptotic cell death induced by chemo-photothermal therapy. Int J Pharm. 2017;531(1):1-3.

95. Maiti D, Saha A, Devi PS. Surface modified multifunctional $\mathrm{ZnFe}_{2} \mathrm{O}_{4}$ nanoparticles for hydrophobic and hydrophilic anti-cancer drug molecule loading. Phys Chem Chem Phys. 2016;18(3):1439-50.

96. Senyei A, Widder K, Czerlinski G. Magnetic guidance of drug-carrying microspheres. J Appl Phys. 1978;49(6):3578-83.

97. Huang J, Li Y, Orza A, Lu Q, Guo P, Wang L, Yang L, Mao H, et al. Magnetic nanoparticle facilitated drug delivery for Cancer therapy with targeted and image-guided approaches. Adv Funct Mater. 2016;26(22):3818-36.

98. Langer R. New methods of drug delivery. Science. 1990;249(4976):1527-33.

99. Raavé $R$, van Kuppevelt TH, Daamen WF. Chemotherapeutic drug delivery by tumoral extracellular matrix targeting. J Control Release. 2018;274:1-8.

100. Pankhurst QA, Connolly J, Jones SK, Dobson J. Applications of magnetic nanoparticles in biomedicine. J Phys D Appl Phys. 2003;36(13):R167.

101. Okon E, Pouliquen D, Okon P, Kovaleva ZV, Stepanova TP, Lavit SG, Kudryavtsev BN, Jallet $P$, et al. Biodegradation of magnetite dextran nanoparticles in the rat. A histologic and biophysical study. Lab Investig. 1994;71(6):895-903.

102. Eivari HA, Rahdar A, Arabi H. Preparation of superparamagnetic iron oxide nanoparticles and investigation their magnetic properties. IJSEl. 2012;1(3):10312-3.

103. Liu C, Rondinone AJ, Zhang ZJ. Synthesis of magnetic spinel ferrite $\mathrm{CoFe}_{2} \mathrm{O}_{4}$ nanoparticles from ferric salt and characterization of the size-dependent superparamagnetic properties. Pure App Chem. 2000;72(1-2):37-45.

104. Yu S, Chow GM. Carboxyl group $\left(-\mathrm{CO}_{2} \mathrm{H}\right)$ functionalized ferrimagnetic iron oxide nanoparticles for potential bio-applications. J Mater Chem. 2004; 14(18):2781-6

105. Weinstein JS, Varallyay CG, Dosa E, Gahramanov S, Hamilton B, Rooney WD, Muldoon LL, Neuwelt EA, et al. Superparamagnetic iron oxide nanoparticles: diagnostic magnetic resonance imaging and potential therapeutic applications in neurooncology and central nervous system inflammatory pathologies, a review. J Cereb Blood Flow Metab. 2010;30(1):15-35.

106. Matuszak J, Lutz B, Sekita A, Zaloga J, Alexiou C, Lyer S, Cicha I, et al. Drug delivery to atherosclerotic plaques using superparamagnetic iron oxide nanoparticles. Int J Nanomedicine. 2018;13:8443.

107. Nikitin A, Khramtsov M, Garanina A, Mogilnikov P, Sviridenkova N, Shchetinin I, Savchenko A, Abakumov M, Majouga A, et al. Synthesis of iron oxide nanorods for enhanced magnetic hyperthermia. J Magn Magn Mater. 2019;469:443-9.

108. Anilkumar TS, Lu YJ, Chen HA, Hsu HL, Jose G, Chen JP, et al. Dual targeted magnetic photosensitive liposomes for photothermal/photodynamic tumor therapy. J Magn Magn Mater. 2019;473:241-52.

109. Jose G, Lu YJ, Chen HA, Hsu HL, Hung JT, Anilkumar TS, Chen JP, et al. Hyaluronic acid modified bubble-generating magnetic liposomes for targeted delivery of doxorubicin. J Magn Magn Mater. 2019;474:355-64. 
110. De Jong WH, Hagens WI, Krystek P, Burger MC, Sips AJ, Geertsma RE, et al. Particle size-dependent organ distribution of gold nanoparticles after intravenous administration. Biomaterials. 2008;29(12):1912-9.

111. Nakamura $H$, Watano $S$. Direct permeation of nanoparticles across cell membrane: a review. KONA Powder and Part J. 2018;35:49-65.

112. Azad TD, Pan J, Connolly ID, Remington A, Wilson CM, Grant GA, et al. Therapeutic strategies to improve drug delivery across the blood-brain barrier. Neurosurg Focus. 2015;38(3):E9.

113. Bruce JN. Improving drug delivery to Intracerebral tumor and surrounding brain in a rodent model: a comparison of osmotic versus Bradykinin modification of the blood-brain and/or blood-tumor barriers. Neurosurgery. 1998:43(4):889.

114. Steichen SD, Caldorera-Moore M, Peppas NA. A review of current nanoparticle and targeting moieties for the delivery of cancer therapeutics. Eur J Pharm Sci. 2013;48(3):416-27.

115. Redhead HM, Davis SS, Illum L. Drug delivery in poly(lactide-co-glycolide) nanoparticles surface modified with poloxamer 407 and poloxamine 908:in vitro characterisation and in vivo evaluation. J Control Release. 2001;70(3): 353-63.

116. Champion JA, Katare YK, Mitragotri S. Particle shape: a new design parameter for micro-and nanoscale drug delivery carriers. J Control Release. 2007;121(1-2):3-9.

117. Liu Y, Tan J, Thomas A, Ou-Yang D, Muzykantov VR. The shape of things to come: importance of design in nanotechnology for drug delivery. Ther Deliv. 2012;3(2):181-94.

118. Mathaes R, Winter G, Besheer A, Engert J. Non-spherical micro-and nanoparticles: fabrication, characterization and drug delivery applications. Expert Opin Drug Deliv. 2015;12(3):481-92

119. Eliezar J, Scarano W, Boase NR, Thurecht KJ, Stenzel MH. In vivo evaluation of folate decorated cross-linked micelles for the delivery of platinum anticancer drugs. Biomacromolecules. 2015;16(2):515-23.

120. MacDonald IC, Schmidt EE, Groom AC. The high splenic hematocrit: a rheological consequence of red cell flow through the reticular meshwork. Microvasc Res. 1991;42(1):60-76.

121. Canelas DA, Herlihy KP, DeSimone JM. Top-down particle fabrication: control of size and shape for diagnostic imaging and drug delivery. Wiley Interdiscip Rev Nanomed Nanobiotechnol. 2009;1(4):391-404.

122. Decuzzi $P$, Pasqualini $R$, Arap W, Ferrari M. Intravascular delivery of particulate systems: does geometry really matter? Pharm Res. 2009;26(1):235.

123. Yoo JW, Doshi N, Mitragotri S. Adaptive micro and nanoparticles: temporal control over carrier properties to facilitate drug delivery. Adv Drug Deliv Rev. 2011;63(14-15):1247-56.

124. Teja AS, Koh PY. Synthesis, properties, and applications of magnetic iron oxide nanoparticles. Prog Cryst Growth Charact Mater. 2009;55(1-2):22-45.

125. Nedyalkova M, Donkova B, Romanova J, Tzvetkov G, Madurga S, Simeonov V. Iron oxide nanoparticles-in vivo/in vitro biomedical applications and in silico studies. Adv Colloid Interface Sci. 2017;249:192-212.

126. Ji X, Peng F, Zhong Y, Su Y, He Y. Fluorescent quantum dots: synthesis, biomedical optical imaging, and biosafety assessment. Colloids Surf B Biointerfaces. 2014;124:132-9

127. Sperling RA, Parak WJ. Surface modification, functionalization and bioconjugation of colloidal inorganic nanoparticles. Philos Trans A Math Phys Eng Sci. 2010;368(1915):1333-83.

128. Zanganeh S, Spitler R, Erfanzadeh M, Alkilany AM, Mahmoudi M. Protein corona: opportunities and challenges. Int J Biochem Cell Biol. 2016;75:143-7.

129. Mitragotri S, Lahann J. Physical approaches to biomaterial design. Nat Mater. 2009;8(1):15-23

130. Lázaro IA, Haddad S, Sacca S, Orellana-Tavra C, Fairen-Jimenez D, Forgan RS, et al. Selective surface PEGylation of UiO-66 nanoparticles for enhanced stability, cell uptake, and pH-responsive drug delivery. Chem. 2017;2(4):561-78.

131. Park J, Wang ZU, Sun LB, Chen YP, Zhou HC. Introduction of functionalized mesopores to metal-organic frameworks via metal-ligand-fragment coassembly. J Am Chem Soc. 2012;134(49):20110-6.

132. Reis CP, Neufeld RJ, Ribeiro AJ, Veiga F. Nanoencapsulation I. Methods for preparation of drug-loaded polymeric nanoparticles. Nanomedicine. 2006; 2(1):8-21.

133. Kreuter JO, Speiser PP. New adjuvants on a polymethylmethacrylate base. Infect Immun. 1976;13(1):204-10.

134. Barichello JM, Morishita M, Takayama K, Nagai T. Encapsulation of hydrophilic and lipophilic drugs in PLGA nanoparticles by the nanoprecipitation method. Drug Dev Ind Pharm. 1999;25(4):471-6.
135. Narvekar M, Xue HY, Eoh JY, Wong HL. Nanocarrier for poorly water-soluble anticancer drugs-barriers of translation and solutions. AAPS PharmSciTech. 2014;15(4):822-33.

136. Loftsson T, Brewster ME. Pharmaceutical applications of cyclodextrins: basic science and product development. J Pharm Pharmacol. 2010;62(11):1607-21.

137. Ismael GF, Rosa DD, Mano MS, Awada A. Novel cytotoxic drugs: old challenges, new solutions. Cancer Treat Rev. 2008;34(1):81-91.

138. Wilczewska AZ, Niemirowicz K, Markiewicz KH, Car H. Nanoparticles as drug delivery systems. PR. 2012;64(5):1020-37.

139. Vignaroli G, Calandro P, Zamperini C, Coniglio F, lovenitti G, Tavanti M, Colecchia D, Dreassi E, Valoti M, Schenone S, Chiariello M, et al. Improvement of pyrazolo [3, 4-d] pyrimidines pharmacokinetic properties: nanosystem approaches for drug delivery. Sci Rep. 2016;6:21509.

140. Liu X, Sun M, Sun J, Hu J, Wang Z, Guo J, Gao W, et al. Polymerization induced self-assembly of a site-specific interferon a-block copolymer conjugate into micelles with remarkably enhanced pharmacology. J Am Chem Soc. 2018;140(33):10435-8.

141. Tian Y, Shi C, Sun Y, Zhu C, Sun CC, Mao S, et al. Designing micellar nanocarriers with improved drug loading and stability based on solubility parameter. Mol Pharm. 2015;12(3):816-25.

142. Cho K, Wang XU, Nie S, Shin DM. Therapeutic nanoparticles for drug delivery in cancer. Clin Cancer Res. 2008;14(5):1310-6.

143. Fortuna A, Alves $G$, Falcao A. In vitro and in vivo relevance of the Pglycoprotein probe substrates in drug discovery and development: focus on rhodamine 123, digoxin and talinolol. J Bioequiv Availab. 2011;1:1-23.

144. Kreuter J. Nanoparticulate systems for brain delivery of drugs. Adv Drug Deliv Rev. 2001;47(1):65-81.

145. Singh R, Lillard JW Jr. Nanoparticle-based targeted drug delivery. Exp Mol Pathol. 2009;86(3):215-23.

146. Maeda H, Wu J, Sawa T, Matsumura Y, Hori K. Tumor vascular permeability and the EPR effect in macromolecular therapeutics: a review. J Control Release. 2000:65(1-2):271-84.

147. Seki T, Fang J. Maeda H. tumor-targeted macromolecular drug delivery based on the enhanced permeability and retention effect in solid tumor. In: Lu Y, Mahato R, editors. Pharmaceutical Perspectives of Cancer Therapeutics. New York: Springer; 2009. p. 93-120.

148. Fang J, Nakamura $H$, Maeda $H$. The EPR effect: unique features of tumor blood vessels for drug delivery, factors involved, and limitations and augmentation of the effect. Adv Drug Deliv Rev. 2011;63(3):136-51.

149. Yin $H$, Liao L, Fang J. Enhanced permeability and retention (EPR) effect based tumor targeting: the concept, application and prospect. JSM Clin Oncol Res. 2014;2(1):1010.

150. Patel B, Kirkwood AA, Dey A, Marks DI, McMillan AK, Menne TF, Micklewright L, Patrick P, Purnell S, Rowntree CJ, Smith P, et al. Pegylated-asparaginase during induction therapy for adult acute lymphoblastic leukaemia: toxicity data from the UKALL14 trial. Leukemia. 2017;31(1):58.

151. Gradishar WJ, Tjulandin S, Davidson N, Shaw H, Desai N, Bhar P, Hawkins M, O'Shaughnessy J, et al. Phase III trial of nanoparticle albumin-bound paclitaxel compared with polyethylated castor oil-based paclitaxel in women with breast cancer. J Clin Oncol. 2005;23(31):7794-803.

152. Desai N, Trieu V, Yao Z, Louie L, Ci S, Yang A, Tao C, De T, Beals B, Dykes D, Noker $P$, et al. Increased antitumor activity, intratumor paclitaxel concentrations, and endothelial cell transport of cremophor-free, albuminbound paclitaxel, ABI-007, compared with cremophor-based paclitaxel. Clin Cancer Res. 2006;12(4):1317-24.

153. Peer D, Karp JM, Hong S, Farokhzad OC, Margalit R, Langer R. Nanocarriers as an emerging platform for cancer therapy. Nat Nanotechnol. 2007;2(12):751.

154. Wicki A, Witzigmann D, Balasubramanian V, Huwyler J. Nanomedicine in cancer therapy: challenges, opportunities, and clinical applications. J Control Release. 2015:200:138-57.

155. Brown MB, Martin GP, Jones SA, Akomeah FK. Dermal and transdermal drug delivery systems: current and future prospects. Drug Deliv. 2006; 13(3):175-87.

156. Parveen S, Misra R, Sahoo SK. Nanoparticles: a boon to drug delivery, therapeutics, diagnostics and imaging. Nanomedicine. 2012;8(2):147-66.

157. Siwowska K, Schmid RM, Cohrs S, Schibli R, Müller C. Folate receptorpositive gynecological cancer cells: In vitro and in vivo characterization. Pharmaceuticals. 2017;10(3):72

158. Shi C, Zhang Z, Wang F, Luan Y. Active-targeting docetaxel-loaded mixed micelles for enhancing antitumor efficacy. J Mol Liq. 2018;264:172-8. 
159. Huang $G$, Huang $H$. Hyaluronic acid-based biopharmaceutical delivery and tumor-targeted drug delivery system. J Control Release. 2018;278:122-6.

160. Lee H, Lee K, Park TG. Hyaluronic acid-paclitaxel conjugate micelles: synthesis, characterization, and antitumor activity. Bioconjug Chem. 2008; 19(6):1319-25

161. Tu Y, Zhu L. Matrix metalloproteinase-sensitive Nanocarriers. In: Smart Pharmaceutical Nanocarriers; 2016. p. 83-116.

162. Dai Z, Yao Q, Zhu L. MMP2-sensitive PEG-lipid copolymers: a new type of tumor-targeted P-glycoprotein inhibitor. ACS Appl Mater Interfaces. 2016; 8(20):12661-73.

163. Mohanraj VJ, Chen Y. Nanoparticles-a review. TJPR. 2006;5(1):561-73.

164. Singh S, Pandey VK, Tewari RP, Agarwal V. Nanoparticle based drug delivery system: advantages and applications. Indian J Sci Technol. 2011;4(3):177-80.

165. Prow TW, Grice JE, Lin LL, Faye R, Butler M, Becker W, Wurm EM, Yoong C, Robertson TA, Soyer HP, Roberts MS, et al. Nanoparticles and microparticles for skin drug delivery. Adv Drug Deliv Rev. 2011;63(6):470-91.

166. Menon GK. New insights into skin structure: scratching the surface. Adv Drug Deliv Rev. 2002;54:S3-17.

167. Silva GA. Introduction to nanotechnology and its applications to medicine. Surg Neurol Int. 2004;61(3):216-20.

168. Neubert RH. Potentials of new nanocarriers for dermal and transdermal drug delivery. Eur J Pharm Biopharm. 2011;77(1):1-2.

169. Desai P, Patlolla RR, Singh M. Interaction of nanoparticles and cellpenetrating peptides with skin for transdermal drug delivery. Mol Membr Biol. 2010;27(7):247-59.

170. Lademann J, Richter H, Teichmann A, Otberg N, Blume-Peytavi U, Luengo J, Weiss B, Schaefer UF, Lehr CM, Wepf R, Sterry W. Nanoparticles-an efficient carrier for drug delivery into the hair follicles. Eur J Pharm Biopharm. 2007; 66(2):159-64.

171. Alvarez-Román R, Naik A, Kalia YN, Guy RH, Fessi H. Skin penetration and distribution of polymeric nanoparticles. J Control Release. 2004;99(1):53-62.

172. Kumar R, Philip A. Modified transdermal technologies: Breaking the barriers of drug permeation via the skin. TJPR. 2007;6(1):633-44

173. Baroli B. Penetration of nanoparticles and nanomaterials in the skin: fiction or reality? J Pharm Sci. 2010;99(1):21-50.

174. Kraft JC, Freeling JP, Wang Z, Ho RJ. Emerging research and clinical development trends of liposome and lipid nanoparticle drug delivery systems. J Pharm Sci. 2014;103(1):29-52.

175. Blade J, Sonneveld P, San Miguel JF, Sutherland HJ, Hajek R, Nagler A, et al. Efficacy and safety of pegylated liposomal doxorubicin in combination with bortezomib for multiple myeloma: effects of adverse prognostic factors on outcome. Clin Lymphoma Myeloma Leuk. 2011;11:44-9.

176. Barenholz Y. Doxil(R)--the first FDA-approved nano-drug: lessons learned. J Control Release. 2012;160:117-34.

177. Mathur V, Satrawala Y, Rajput M. Physical and chemical penetration enhancers in transdermal drug delivery system. Asian J Pharm. 2010; 4(3):173.

178. Moghassemi S, Hadjizadeh A. Nano-niosomes as nanoscale drug delivery systems: an illustrated review. J Control Release. 2014;185:22-36.

179. Muzzalupo R, Tavano L, Cassano R, Trombino S, Ferrarelli T, Picci N, et al. A new approach for the evaluation of niosomes as effective transdermal drug delivery systems. Eur J Pharm Biopharm. 2011;79(1):28-35.

180. Fan G, Xu Z, Liu X. Preparation of pomegranate Ellagic acid inclusion complex gel and its transdermal permeation in vitro. Procedia Eng. 2017; 174:724-31.

181. Junyaprasert VB, Singhsa P, Jintapattanakit A. Influence of chemical penetration enhancers on skin permeability of ellagic acid-loaded niosomes. Asian J Pharm. 2013;8(2):110-7.

182. Barua S, Mitragotri S. Challenges associated with penetration of nanoparticles across cell and tissue barriers: a review of current status and future prospects. Nano Today. 2014;9(2):223-43.

183. Thakur K, Geeta A, Kumar Hari SL. Nanocarrier for transdermal drug delivery systems. World J Pharm Pharm Sci. 2016;5:708-23.

184. Shakeel F, Baboota S, Ahuja A, Ali J, Aqil M, Shafiq S, et al. Nanoemulsions as vehicles for transdermal delivery of aceclofenac. AAPS PharmSciTech. 2007;8(4):191.

185. Hooper JW, Golden JW, Ferro AM, King AD. Smallpox DNA vaccine delivered by novel skin electroporation device protects mice against intranasal poxvirus challenge. Vaccine. 2007;25(10):1814-23.

186. Bramson J, Dayball K, Evelegh C, Wan YH, Page D, Smith A, et al. Enabling topical immunization via microporation: a novel method for pain-free and needle-free delivery of adenovirus-based vaccines. Gene Ther. 2003;10(3):251.

187. Prausnitz MR, Langer R. Transdermal drug delivery. Nature Biotechnol. 2008; 26(11):1261-8

188. Lee H, Song C, Baik S, Kim D, Hyeon T, Kim DH, et al. Device-assisted transdermal drug delivery. Adv Drug Deliv Rev. 2017;127:35-45.

189. Münch S, Wohlrab J, Neubert RH. Dermal and transdermal delivery of pharmaceutically relevant macromolecules. Eur J Pharm Biopharm. 2017;119: 235-42.

190. Chen Y, Liu L. Modern methods for delivery of drugs across the blood-brain barrier. Adv Drug Deliv Rev. 2012;64(7):640-65.

191. Bicker J, Alves G, Fortuna A, Falcão A. Blood-brain barrier models and their relevance for a successful development of CNS drug delivery systems: a review. Eur J Pharm Biopharm. 2014;87(3):409-32.

192. Abbott NJ, Rönnbäck L, Hansson E. Astrocyte-endothelial interactions at the blood-brain barrier. Nat Rev Neurosci. 2006;7(1):41-53.

193. Alyautdin R, Khalin I, Nafeeza MI, Haron MH, Kuznetsov D, et al. Nanoscale drug delivery systems and the blood-brain barrier. Int J Nanomedicine. 2014:9:795-811.

194. El-Bachá RD, Minn A. Drug metabolizing enzymes in cerebrovascula endothelial cells afford a metabolic protection to the brain. Cell Mol Biol. 1999;45(1):15-23.

195. Alyautdin R, Gothier D, Petrov V, Kharkevich D, Kreuter J. Analgesic activity of the hexapeptidedalargin adsorbed on the surface of polysorbate 80coated poly(butyl cyanoacrylate) nanoparticles. Eur J Pharm Biopharm. 1995; 41(1):44-8.

196. Qiao R, Jia Q, Hüwel S, Xia R, Liu T, Gao F, Galla HJ, Gao M, et al. Receptormediated delivery of magnetic nanoparticles across the blood-brain barrier. ACS Nano. 2012;6(4):3304-10.

197. Posadas I, Monteagudo S, Ceña V. Nanoparticles for brain-specific drug and genetic material delivery, imaging and diagnosis. Nanomedicine. 2016;11(7): 833-49.

198. Masserini M. Nanoparticles for brain drug delivery. ISRN Biochem. 2013;2013: 238428

199. Gao H, Pang Z, Jiang X. Targeted delivery of nano-therapeutics for major disorders of the central nervous system. Pharm Res. 2013;30(10):2485-98.

200. Hwang SR, Kim K. Nano-enabled delivery systems across the blood-brain barrier. Arch Pharm Res. 2014:37(1):24-30.

201. Wei X, Chen X, Ying M, Lu W. Brain tumor-targeted drug delivery strategies. Acta Pharm Sin B. 2014:4(3):193-201.

202. Lu CT, Zhao YZ, Wong HL, Cai J, Peng L, Tian XQ, et al. Current approaches to enhance CNS delivery of drugs across the brain barriers. Int J Nanomedicine. 2014;9:2241-57.

203. Groothuis DR. The blood-brain and blood-tumor barriers: a review of strategies for increasing drug delivery. Neuro-Oncology. 2000;2(1):45-59.

204. Charlton S, Jones NS, Davis SS, Illum L. Distribution and clearance of bioadhesive formulations from the olfactory region in man: effect of polymer type and nasal delivery device. Eur J Pharm Biopharm. 2007;30(34):295-302.

205. Dhuria SV, Hanson LR, Frey WH. Intranasal delivery to the central nervous system: mechanisms and experimental considerations. J Pharm Sci. 2010; 99(4):1654-73.

206. Das S, Chaudhury A. Recent advances in lipid nanoparticle formulations with solid matrix for oral drug delivery. AAPS PharmSci Tech. 2011;12(1) 62-76.

207. Plapied L, Duhem N. des Rieux A, Préat V. Fate of polymeric nanocarriers for oral drug delivery. Curr Opin Colloid Interface Sci. 2011;16(3):228-37.

208. Tan ML, Choong PF, Dass CR. Recent developments in liposomes, microparticles and nanoparticles for protein and peptide drug delivery. Peptides. 2010;31(1):184-93.

209. Shantha Kumar TR, Soppimath K, Nachaegari SK. Novel delivery technologies for protein and peptide therapeutics. Curr Pharm Biotechnol. 2006;7(4):261-76.

210. Allémann E, Leroux JC, Gurny R. Polymeric nano-and microparticles for the oral delivery of peptides and peptidomimetics. Adv Drug Deliv Rev. 1998; 34(2-3):171-89.

211. Agrawal U, Sharma R, Gupta M, Vyas SP. Is nanotechnology a boon for oral drug delivery? Drug Discov Today. 2014:19(10):1530-46.

212. Ensign LM, Cone R, Hanes J. Oral drug delivery with polymeric nanoparticles: the gastrointestinal mucus barriers. Adv Drug Deliv Rev. 2012; 64(6):557-70 
213. Makhlof A, Tozuka Y, Takeuchi H. Design and evaluation of novel pHsensitive chitosan nanoparticles for oral insulin delivery. Eur J Pharm Sci. 2011;42(5):445-51.

214. Kim JH, Kim YS, Park K, Kang E, Lee S, Nam HY, Kim K, Park JH, Chi DY, Park RW, Kim IS, et al. Self-assembled glycol chitosan nanoparticles for the sustained and prolonged delivery of antiangiogenic small peptide drugs in cancer therapy. Biomaterials. 2008;29(12):1920-30.

215. Pridgen EM, Alexis F, Farokhzad OC. Polymeric nanoparticle technologies for oral drug delivery. Clin Gastroenterol Hepatol. 2014;12(10):1605-10.

216. Morishita M, Peppas NA. Is the oral route possible for peptide and protein drug delivery? Drug Discov Today. 2006;11(19-20):905-10.

217. Zhang Z, Shan H, Chen L, He C, Zhuang $X$, Chen $X$, et al. Synthesis of pHresponsive starch nanoparticles grafted poly(l-glutamic acid) for insulin controlled release. Eur Polym J. 2013;49(8):2082-91.

218. Cha S, Lee SH, Kang SH, Hasan MN, Kim YJ, Cho S, Lee YK, et al. Antibodymediated oral delivery of therapeutic DNA for type 2 diabetes mellitus. Biomater Res. 2018;22(1):19.

219. Kumar P, Sharma G, Gupta V, Kaur R, Thakur K, Malik R, Kumar A, Kaushal N, Katare OP, Raza K, et al. Oral delivery of Methylthioadenosine to the brain employing solid lipid nanoparticles: pharmacokinetic, behavioral, and Histopathological evidences. AAPS Pharm Sci Tech. 2019;20(2):74.

220. Islam MS, Reineke J, Kaushik RS, Woyengo T, Baride A, Alqahtani MS, Perumal O, et al. Bioadhesive Food-Protein Nanoparticles as Pediatric Oral Drug Delivery system. ACS Appl Mater Interfaces. 2019;11:18062-73.

221. Yang J, Guo X, Fu C, Zhao S, Li J, Zhou Y, Huang L, Li Y, Xu J, et al. Lipid nanoparticles-encapsulated YF4: a potential therapeutic Oral peptide delivery system for hypertension treatment. Front Pharmacol. 2019;10:102.

222. Paranjpe M, Müller-Goymann CC. Nanoparticle-mediated pulmonary drug delivery: a review. Int J Mol Sci. 2014;15(4):5852-73.

223. Park CW, Mansour HM, Hayes D. Pulmonary inhalation aerosols for targeted antibiotics drug delivery. European Pharmaceutical Review. 2011;16(1):32-6.

224. Shoyele SA, Slowey A. Prospects of formulating proteins/peptides as aerosols for pulmonary drug delivery. Int J Pharm. 2006;314(1):1-8.

225. Mangal S, Gao W, Li T, Zhou QT. Pulmonary delivery of nanoparticle chemotherapy for the treatment of lung cancers: challenges and opportunities. Acta Pharmacol Sin. 2017;38(6):782.

226. Xu C, Wang Y, Guo Z, Chen J, Lin L, Wu J, Tian H, Chen X. Pulmonary delivery by exploiting doxorubicin and cisplatin co-loaded nanoparticles for metastatic lung cancer therapy. J Control Release. 2019;295:153-63.

227. LiCalsi C, Christensen T, Bennett JV, Phillips E, Witham C. Dry powder inhalation as a potential delivery method for vaccines. Vaccine. 1999;17(1314):1796-803.

228. Lu D, Hickey AJ. Pulmonary vaccine delivery. Expert Rev Vaccines. 2007; $6(2): 213$

229. Weibel ER. Geometric and dimensional airway models of conductive, transitory and respiratory zones of the human lung. In: Morphometry of the human lung springer. Berlin: Heidelberg; 1963. p. 136-42.

230. Yhee JY, Im J, Nho RS. Advanced therapeutic strategies for chronic lung disease using nanoparticle-based drug delivery. J Clin Med. 2016:5(9):82.

231. Patton JS, Byron PR. Inhaling medicines: delivering drugs to the body through the lungs. Nat Rev Drug Discov. 2007;6(1):67.

232. Kaminskas LM, McLeod VM, Ryan GM, Kelly BD, Haynes JM, Williamson M, Thienthong N, Owen DJ, Porter CJ, et al. Pulmonary administration of a doxorubicin-conjugated dendrimer enhances drug exposure to lung metastases and improves cancer therapy. J Control Release. 2014;183:18-26.

233. El-Sherbiny IM, El-Baz NM, Yacoub MH. Inhaled nano-and microparticles for drug delivery. Glob Cardiol Sci Pract. 2015;1:2.

234. Schürch S, Gehr P, Im Hof V, Geiser M, Green F. Surfactant displaces particles toward the epithelium in airways and alveoli. Respir Physiol. 1990;80(1):17-32.

235. Geiser M, Rothen-Rutishauser B, Kapp N, Schürch S, Kreyling W, Schulz H, Semmler M, Im Hof V, Heyder J, Gehr P, et al. Ultrafine particles cross cellular membranes by nonphagocytic mechanisms in lungs and in cultured cells. EHP. 2005;113(11):1555.

236. Carvalho TC, Peters JI, Williams RO III. Influence of particle size on regional lung deposition-what evidence is there? Int J Pharm. 2011;406(1-2):1-10.

237. Byron PR. Prediction of drug residence times in regions of the human respiratory tract following aerosol inhalation. J Pharm Sci. 1986;75(5):433-8.

238. Ryan GM, Kaminskas LM, Kelly BD, Owen DJ, McIntosh MP, Porter CJ, et al. Pulmonary administration of PEGylatedpolylysinedendrimers: absorption from the lung versus retention within the lung is highly size-dependent. Mol Pharm. 2013;10(8):2986-95.
239. Harris JM, Chess RB. Effect of pegylation on pharmaceuticals. Nat Rev Drug Discov. 2003;2(3):214

240. Grotz E, Tateosian NL, Salgueiro J, Bernabeu E, Gonzalez L, Manca ML, Amiano N, Valenti D, Manconi M, García V, Moretton MA, et al. Pulmonary delivery of rifampicin-loaded soluplus micelles against Mycobacterium tuberculosis. J Drug Deliv Sci Technol. 2019;53:101170.

241. Huang Z, Huang Y, Ma C, Ma X, Zhang X, Lin L, Zhao Z, Pan X, Wu C, et al. Endotracheal Aerosolization device for laboratory investigation of pulmonary delivery of nanoparticle suspensions: in vitro and in vivo validation. Mol Pharm. 2018;15(12):5521-33.

242. Mohamed A, Pekoz AY, Ross K, Hutcheon GA, Saleem IY. Pulmonary Delivery of Nanocomposite Microparticles (NCMPs) incorporating miR-146a for Treatment of COPD. Int J Pharm. 2019;569:118524.

243. Zhang Y, He RQ, Wang X, Chen YR, Li MW, Zhang XL, Ma J, Chen G, Hu XH, et al. IRAK1 and TRAF6, inversely modulated by antitumor miR-146a-5p, markedly promotes the progression of NSCLC. Int J Clin Exp Pathol. 2017; 10(5):4955-68.

244. Parker SE, Davey PG. Pharmacoeconomics of intravenous drug administration. Pharmacoeconomics. 1992;1(2):103-15.

245. Kalepu S, Nekkanti V. Insoluble drug delivery strategies: review of recent advances and business prospects. Acta Pharm Sin B. 2015;5(5):442-53.

246. Wong J, Brugger A, Khare A, Chaubal M, Papadopoulos P, Rabinow B, Kipp J, Ning J, et al. Suspensions for intravenous (IV) injection: a review of development, preclinical and clinical aspects. Adv Drug Deliv Rev. 2008; 60(8):939-54.

247. Sasikumar A, Kamalasanan K. Nanomedicine for prostate cancer using nanoemulsion: a review. J Control Release. 2017;260:111-23.

248. Dias ML, Carvalho JP, Rodrigues DG, Graziani SR, Maranhão RC. Pharmacokinetics and tumor uptake of a derivatized form of paclitaxel associated to a cholesterol-rich nanoemulsion (LDE) in patients with gynecologic cancers. Cancer Chemother Pharmacol. 2007;59(1):105-11.

249. Wei $Y$, Zhao L. Passive lung-targeted drug delivery systems via intravenous administration. Pharm Dev Techno. 2014;19(2):129-36.

250. Hörmann K, Zimmer A. Drug delivery and drug targeting with parenteral lipid nanoemulsions - a review. J Control Release. 2016;223:85-98.

251. Cooke M, Lamplugh A, Naudeer S, Edey M, Bhandari S. Efficacy and tolerability of accelerated-dose low-molecular-weight iron dextran (Cosmofer) in patients with chronic kidney disease. Am J Nephrol. 2012; 35(1):69-74.

252. Pai AB. Complexity of intravenous iron nanoparticle formulations: implications for bioequivalence evaluation. Ann N Y Acad Sci. 2017; 1407(1):17-25.

253. Wang G, Gao S, Tian R, Miller-Kleinhenz J, Qin Z, Liu T, Li L, Zhang F, Ma Q, Zhu $\mathrm{L}$, et al. Theranostic hyaluronic acid-Iron Micellar nanoparticles for magnetic-field- enhanced in vivo Cancer chemotherapy. Chem Med Chem. 2018;13(1):78-86.

254. Gnach A, Lipinski T, Bednarkiewicz A, Rybka J, Capobianco JA. Upconverting nanoparticles: assessing the toxicity. Chem Soc Rev. 2015;44(6):1561-84.

255. Bencsik A, Lestaevel P, Canu IG. Nano-and neurotoxicology: an emerging discipline. Prog Neurobiol. 2017;160:45-63.

256. Khanna P, Ong C, Bay BH, Baeg GH. Nanotoxicity: an interplay of oxidative stress, inflammation and cell death. Nanomaterials. 2015;5(3):1163-80.

257. Albanese A, Tang PS, Chan WC. The effect of nanoparticle size, shape, and surface chemistry on biological systems. Annu Rev Biomed Eng. 2012;14:1-6.

\section{Publisher's Note}

Springer Nature remains neutral with regard to jurisdictional claims in published maps and institutional affiliations. 